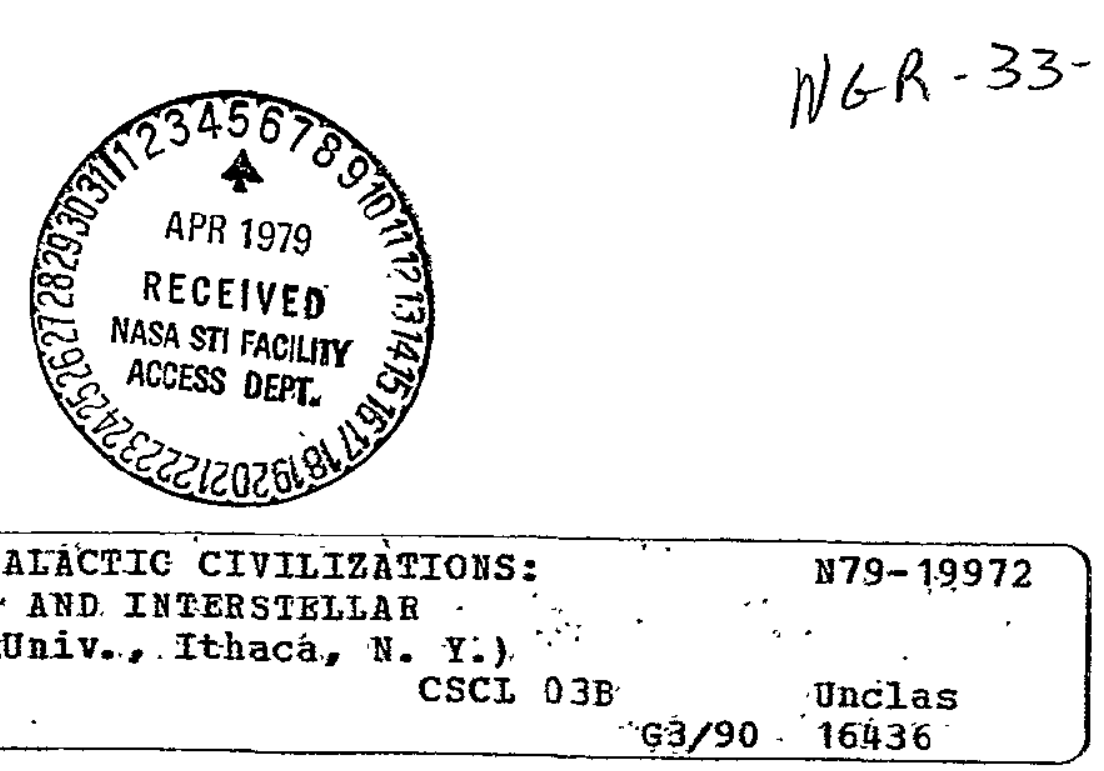

(NASA-CR-158245) - GATACPIC CIVILIZATIONS : POPUI,ATION DYNAMICS AND. INTERSTELIAR -

DIFFusION (Corneli Univ.., Ithaca, N. Y:)

90 :p: HC. A05/HF .A01

CSCL $03 \mathrm{~B}$ 16436

\title{
CORNELL UNIVERSITY Center for Radiophysics and Space Research.
}

ITHACA, N. Y.

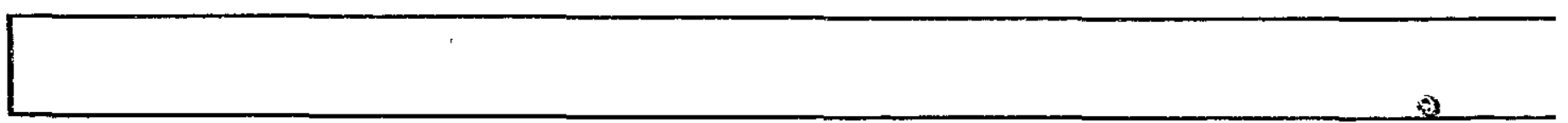

CRSF 711

GALACTIC CIVILIZATIONS: POPULATION DYNAMICS AND

INTERSTELIAR DIEFUSTON

by

William I. Newman and CarI Sagan 
GALACTIC CIVILIZATIONS: POPULATION DYNAMICS AND INTERSTELIAAR DIFFUSION

\author{
by \\ William I. Newman* and Carl Sagan \\ Laboratory for Planetary Studies \\ Cornell University. \\ Ithaca, New York 14853 \\ Decémber 1978
}

*Present Address: Institute for Advanced Study

Princeton, N.J. 08540 


\section{ABSTRACT}

The interstellar diffusion of galactic civilizations is reexamined by potential theory; both numerical and analytical solutions are derived for the non-linear partial differential equations which specify a range of relevant models, drawn from blast wave physics, soil science, and, especially, population biology. An essential feature of these models is that, for all civilizations, population growth must be limited by the carrying capacity of the environment. Dispersal is fundamentally a diffusion process; a eensity-dependent diffusivity describes interstellar emigration. We concentrate on two models, the first describing zero population growth (ZPG) and the second which also ircludes local growth and saturation of a planetary population, and for which we find an asymptotic travel-. -Iing wave solution, For both models the colonization wavefront' expands slowly and uniformly but only the frontier worlds are sources of. further expansion. For nonlinear diffusion with growth and saturation, the colonization wavefront from the nearest independently-arisen galactic civilization can have reached the Earth only if its lifetime exceeds $3 \times 10^{7} \mathrm{yrs}$. For ZPG the corresponding number is $1.3 \times 10^{10} \mathrm{yrs}$. For these numerical results depend on our choices for the specific emigration rate, the distribution of colonizable worlds, and, in the second model, the population growth rate; but the dependences on these parameters is entrancingly weak. We conclude that the Earth is uncolonized not because interstellar spacefaring societies are rare, but because.there are too many worlds to be conquered in the plausible lifetimes of the colonization 
phase of nearby galactic civilizations. However, the radar and television announcement of an emerging technical civilization on Earth may cause a rapid response by nearby civilizations, presently motivated to reach our system directly, rather than by random diffusion . 
Alexander wept when he heard from Anaxarchus chat there was an infinice number of worlds; and his friends asking him if anjy accident had befallen him, he returned this answer: "Do you not think. it a matter worthy of lamentation that when there is such a vast multitude of them, we have not yet conquered one?"

Plutarch, On the Tranquility of the Mind

"Where are they?" is a famous and possibly apocryphal guestion posed by Enrico Fermi at Los Alamos in the late 1940s. In a galaxy with $10^{11}$ stars; with planets apparently abundant and the origin of life seemingly requiring very general cosmic circumstances; with the selective advantage of intelligence and technology obvious and with billions of years available for evolution, should not extraterrestrial intelligence be readily detectable? This. question is most often phrased in its astrophysical context (see, for exampie, Shlklovskii and Sagan, 1966; Drson, 1966): should not extraterrestrial civilizations miliions or, perhaps, even billions of years more advanced then our own be capable of so altering cosmic objects and energy sources that a range of astrophysical phenomena should exist which cannot be understood aparc from the hypothesis of intelligent origin? 


$$
\text { -2- CROMAL PAGE }
$$

An answer is sometimes offered in the negative (Shklovskii, 1976); i.e., the apparent absence of astrophysical phenomena of intelligent origin is taken to demonstrate that no very advanced civilizations exist.-- either because there are as yet undetermined impediments to the evolution of technical civilizations or because such civilizations inevitably self-destruct early in their histories. Alternatively, it can be argued (Sagan, 1974) that there are a wide range of poorly understood astrophysical phenomena, some involving extremely high energies, and that (Sagan, 1973a) the manifestacions of very advanced civilizations would be no more apparent to us than the design and function of human engineering artifacts are to ants crawling upon their surfaces. Any astroengineering activity that is so

wasteful of energy as to be observable with our limited technology might, by its very nature, be necessarily shorclived. The fact that we have not yet acquired compelling evidence of such activity since our rise as a technological society -- although there are certainly unexplained major astrophysical phenomena -is not evidence for the absence of extraterrestrial civilizations. It may rather be that we were not looking in the right place, with the right instruments at the right time. In any case, the sole productive approach is the open-minded pursuit of physical explanations of astrophysical phenomena, where only a 
serious failure might be evidence for extraterrestrial intelligence, but where, in our present ignorance, the possibility remains entirely moot.

However, there is another and more modest side to the question. Pioneers 10 and 11 and Voyagers 1 and 2 show that even our infant technical civilization is capable of interstellar spaceflight, although at velocities $n 10^{-4} \mathrm{pc} \mathrm{yr}^{-1}$. Accordingly, should not civilizations only a little more advanced than we be effortlessly plying the spaces between the stars (Sagan, 1963; Shklovskii and Sagan, 1966)? And, if so, should we not on Earth today have some evidence of interstellar visits? Occasional serious attempts to deal with this question always conclude that no persuasive evidence of past visits exists in human legends or artifacts (Shklovskii and Sagan, 1966; Sagan, 1979); and the most widely touted claims of such visits uniformly have another and more plausible explanation --' generally in the area of archaeology or hoax (see, for example, Story, 1976). Likewise, the extraterrestrial hypothesis of UFOs -that is, the contention that we are today being visited -also exhibits, despite a great deal of study, no persuasive evidence (Sagan and Page, 1972; Klass, 1974). These discussions by no means exclude past or present visits to the Earth; they merely stress the absence of strong evidence for such visits. 
If the presence of extraterrestrial civilizations were to imply visits to Earth then the absence of such visits would imply the absence of such civilizations. This argument was put forward by Hart (1975) who advanced it primarily in the context of interstellar colonization, not exploration. He argues that even if there are energetic difficulties or safety hazards (for example, the induced cosmic ray flux) to relativistic interstellar spaceflight, there should be no serious obstacles to interstellar flight ac $0.1 \mathrm{c}$, in which case the Galaxy would be traversed in $<10^{6}$ yr. Other possible objections to làge scale interstellar spacefaring -- such as loss of motivation or self-destruction -- Hart argues are unlikely because they must apply to all galactic civilizations to explain the absence of extraterrestrials on Earth.

We believe Hart's analysis is flawed on a number of counts. On the one hand there are universal social impediments to cosmic imperialism of a sort which should apply to every galactic civilization. Von Hoener (1973) has remarked that even incerstellar colonization at the speed of light cannot solve the present human population explosion on the planer Earth. With our present exponential growth rate of 
$Y=0.02 \mathrm{yr}^{-1}$ and the colonization sphere expanding at the speed of light, in 500 years the expansion volume will have a radius of $50 \mathrm{pc}$ with all habitable planets in that volume reaching the Earth's present population density; thereafter the population growth rate must decline. This is, of course, an extreme example but it demonstrates that every society which is to avoid severe overcrowding and exhaustion of resources must practice stringent population control and actively maintain very small values of $\gamma$. Since, in the long run, exponentials defeat power laws on every planet 
in the Galaxy -- independent of local biology, evolutionary history, and social customs -- any analysis of this problem must consider low values of $\gamma$ and, in the limit, zero popula-. tion growth (ZPG). A similar point has been made by Cox (1976). One of the objectives of the present paper is to explore in some detail the consequences of low values of $\gamma$ for interstellar colonization.

Another possible social impediment to rapid interstellar colonization, with some conceivable claim to universality, is immortality (Kevles, 1975). In extrapolating to advanced technological societies we often seem willing to imagine daring engineering developments such as interstellar spaceflight with velocities $\mathrm{v} \simeq \mathrm{c}$, but make only the most modest extrapolations in biology or psychology. If aging is due, e.g, , to the accumulation of somatic mutations, it is. conceivable that an advanced society will have essentially eliminated both disease and aging and that the limits to individual longevity will then be set by accidents and by events in geological and stellar evolution. The motivations which we consider reasonable may seem very unnatural to such a society. To what extent, for example, is our motive for interstellar 
colonization itself a quest for immortality -- which would be rendered unnecessary if personal immortality existed? A population of immortal organisms, each leaving . a. offspring every year, exhibits a growth rate identical to that of a population of organisms in which each lives for a single year and then dies leaving $(\underline{a}+\underline{1})$ offspring (Cole, 1954). But if there is a selective advantage for immortality, the relative number of offspring of immortals and mortals will adjust themselves in favor of the former. A society of immortals would be required to practice more stringent population control than a society of mortals. In addition, whatever its other charms, interstellar spaceflight must pose more serious hazards than residence on the home planet. To the extent that such predispositions are inherited, natural selection would tend in such a world to eliminate those individuals without a deep passion for the longest possible lifespans, assuming no differential replication between those in favor of and those opposed to individual longevity. The net result might be a civilization with a profound commitment to stasis even on rather long cosmic time scales and a predisposition antithetical to'interstellar colonization. 
With even modest annual growth rates in science and technology it is clear that a civilization $10^{6}$ or $10^{8}$ years in advance of ours would have technological capabilities which for us would be indistinguishable from magice. The possibility exists that such a civilization might impose an absolutely stringent galactic hegemony. The establishment of an unbreakable Codex Galactica imposing strict injunctions against colonization of or contact with already populated planets (Shklovskir and Sagan, 1966, o. 451) is by no means excluded. This idea, which has been called the Zoo Hypothesis by BaII (1973), is not irrelevant to our concerns merely because it is by definition unprovable; the detection, for example by radio astronomy, of civilizations on planets of other worlds would make the hypothesis falsifiable. It is clearly impossible to prognosticate reliably on the social behavior of a hypothetical advanced civilization, but these examples are put forth to suggest that there may be many, still less apparent, social impediments to extensive interstellar colonization.

On the other hand, Hart seems to have made a fatal confusion between the velocity of individual spacecraft and the velocity of a colonization wave front (see also Jones, 1976; Cox, 1976). In the history of human exploration and colonization 
it is not a single exploratory society which launches all such ventures, but colonies of colonies and. higher order descendants. For example, it might be argued that American exploration of the solar system is a fifth-order Phoenician exploratory venture, the Phoenicians having sattled Carthage, the Carthagenians having settled Iberian seaports, the Iberians having discovered America, and the United States exploring the solar system. Lebanon and Tunisia and Spain are not today spacefaring civilizations. There was a waiting time of centuries to millenia before the colony acquired sufficient resources to initiate independent exploracion or colonization, during which period the parent civilization declined. If we imagine the nth order colonization of a. new planet, the time for acquiring an $(n \div 1)$ th order independent colonization capability will be substantially longer.

Jones (1976, 1978) claims to show from a Monte Cario calculation that even allowing for this colonization waiting time it is very difficult to make the colonization wave front move more slowly than about 10\% of the starship velocity - implying that a single expansionist power will colonize che galaxy in i $10^{7}$ years if the starship velocities are $0.1 \mathrm{c}$. Colonization. for Jones provides an escape valve for an overpopulated civilization. Consequently, his colonization front progresses rapidly. 
Since, in his model, the number of colonists at any given time is proportional to the number of "saturated" planets, the asymptotic behavior of his model must be characterized by exponential population growth and by a steadily accelerating colonization front. We believe this conclusion to be in error and below present population dynamics arguments on the velocity $v$ of such interstellar colonial ventures. It is clear that a comprehensive approach to the problem must include considerations of the population growth rates; the related waiting time for the acquisition of an independent colonial capability on a newly colonized planet; the number of colonists which arrive on a virgin world with each expedition (the larger this number the smaller the waiting time until the next order colonial venture); the starship velocities and search strategies; the abundance of untenanted planets available for colonization; the lifetime of the colonial civilization and its descendants; and, not unrelated, the possibility of interaction between two independent expanding colonial wave fronts originating on separate worlds.

We wish to assess the assumption, implicit in previous speculations on this subject, that the colonization wave front advances with an effective velocity comparable to 
that of the individual starships. In our analysis, we adopt some of the methods conventionally employed in describing growth processes and dispersal mechanisms in terrestrial ecosystems. Their mathematical development provides a rigorous description of the stochastic properties of such processes and permits the use of potential theory. Consequently, we can trace the evolution of the distribution of the organisms that are of interest without following the detailed behavior of each of its individual members.

The models we presently describe can be used to simulate the growth, saturation and expansion of an interstellar civilization "in the mean." Since the mathematical formulation of the problem is accomplished via potential theory, our models are completely specified by a family. of nonlinear partial differential equations. The properties of these equations can be examined analytically, as well as numerically, and the results are directly amenable to scaling. To make the discussion entirely clear it will be necessary first to review some elementary arguments. 
In biological applications, these models have proved to be sufficiently general in describing all but the most catastrophic population dynamics phenomena. Moreover, the results they provide in the interstellar context are remarkably insensitive to the detailed description (within limits) of the model and the associated input parameters. Consequently, the solutions obtained below are, we believe, less vulnerable to charges of terrestrial chauvinism than many previous studies.

We now review the population dynamics processes relevant to our discussion, explore various features of their associated mathematical models, and discuss the implications of the model results. The most significant of these is that the expansion velocity of the colonization front is several orders of magnitude smaller than had been previously anticipated. Thus, the answer to the question "Where are they?" may well be that only now are they about to arrive. 
1. Homiogerieotis Processes

It is convenient, first, to consider the population dynamics processes that occur in a homogeneous environment. [In this instance, we ignore the factors that contribute to immigration and emigration, for example in humans. In animal species, biologists describe such movements as "dispersal," a term used to describe all factors that lead to the displacement of an organism from its place of birth to the location at which it reproduces or dies. [See, for example, Howard (1960).] The simplest description of growth is that the time rate of change of a population $\checkmark$ 'is proportional to the population, or

$$
\frac{d v}{d t}=\gamma v
$$

where the growth rate, $\gamma$, is the difference between the birth and death rates. Exponential population growth is often associated with Malthus' name although the idea that populations increase geometrically seems to have evolved in the Middle Ages, if not in classical antiquity. I/

\footnotetext{
1/ Leonardo Pisano (Fibonacci) in the year 1202 attempted Eo reintroduce into Europe the study of algebra. He posed the problem, complicated by overlapping generations, of how rapidly rabbits wotld reproduce from an initial pair in the first month and then a second pair in the second month before becoming infertile. The number of pairs capable of reproducing in a given month corresponds to the Fibonacci series $1,2,3 ; 5,8,13, \therefore$. Asymptotically, the number of fertile rabbits increases by $\left[\left(5^{\frac{2}{2}}+1\right) / 2\right]$ each month.
} 
The human population growth rate has remained relatively uniform, with a numerical value of approximately $0.056 \mathrm{yr}^{-1}$, from the dawn of civilization until the middle of the eighteenth century. Since that time, in a period representing only $0.02 \%$ of human history, the Earth has witnessed a tenfold increase in human numbers. Population experts have speculated that, for the past two centuries, we have been undergoing a demograpinic transition. It began with a decline in the death rate precipitated by advances in public health and nutrition. Later, the birth rate declined, primarily because of changes in the perceived value of having children. In the interim, the growth rate mushroomed and only now shows signs of stabilizing. Malthus in his. Essay on Population, published in 1798, recognized the Iundamental incompatability between a geometrical progression in population and the finite capacity of the environment co support life. He argued that, in the absence of "moral restraint," other fonns of birth control then unknown or considered unconscionable, the combined ravages of war, famine and pestilence would impose a limit to growth.

A conceptually straightforward generalization of (I) which is Irequently employed in describing this situacion is

$$
\frac{d v}{d t}=\gamma v \underline{f}(v)
$$


where $f(v)$ has the properties

$$
f(0)=1 ; d f(v) / d v \leq 0 ; E\left(v_{s}\right)=0
$$

and where $v_{s}$ describes the carrying capacity of the environment as the maximum population that it can support: The function $f(v)$ decreases in a manner that reflects the diminishing ability of a particular environment to support population growth. A simple but representative example of this function is

$$
f(v)= \begin{cases}1-v / v_{s}, & v<v_{s} \\ 0, & v \geq v_{s}\end{cases}
$$

The corresponding differential equation for the population growth (the Pearl-Verhulst law)

$$
\frac{d v}{d t}=\gamma v\left(1-v / v_{s}\right)
$$

has a general solution given by

$$
v(t)=\frac{v(0) v_{s} e^{\gamma t}}{\left[v_{s}-v(0)\right]+v(0) e^{\gamma t}}
$$

where $v(0)$ is the initial value of $v(t)$. This curve has a characteristic $S$ shape and is referred to as the logistic. 
This curve shares the qualitative properties common to virtually all models of homogeneous processes: exponential (Malthusian) growth for $\nu \ll v_{s}$, followed asymptotically by saturation at $\nu_{s}$.

We employ the pearl-Verhulst

law extensively in what follows to describe homogeneous processes. A particularly readable account of the appropriate nathematical theory (as well as. equations describing multispecies interaction or "predator-prey" systems) may be found in Davis (1962). Coale (1974) provides an interesting introduction to the history of the human population.

\section{Heterogeneous Processes}

Many factors contribute to the rates of dispersal in animal species. Certain ones are innate; others are environmental in origin (Howard, 1960). The simplest of these, random dispersal, is a mechanism that evolved in order to reduce the incidence of homozygotes. An animal such as the muskrat; Ondatra zibethica $I$., will head off in a randomly selected direction over some characteristic distance before it mates, reproduces and dies. Since inbreeding is in this way avoided, the frequency of generic recombination. of deleterious recessive mutations is reduced. 
From a probabilistic point of view, each muskrat executes (in the absence of population growth) a random walk or Brownian motion: On the other hand, the behavior of the muskrat population distribution is described by. the diffusion equation.

The equivalence of Brownian motion and diffusion is well-known (see, for example, Chandrasekhar, 1943; or Karlin and Taylor, 1975). Since a firm understanding of these. phenomena is essential for our discussion, and has been incompletely appreciated in some previous discussions of the problem, we consider a simple and standard example -- the drunkard"s walk. We have a linear array of lampposts, each a distance $l$ from the next, that lights the street on which both a bar and a drunkard's home are situated. The drunkard is able only to walk the distance between one post and the next before he collapses. He then arises a time $\tau$ later and sets out toward another lamppost. The time intervel bectiven consecutive drunkard-Iamppost collisions is then $\tau$ : Unfortunately, he has no sense of direction and it is equally likely that he will stagger to the left as to the right. If $o_{n}^{k}$ is the probability that he is. at lampoost $n$ at time interval $k$, then the equation governing his behavior is readily observed to be

$$
0_{n}^{k+1}=\frac{1}{2} 0_{n+1}^{k}+\frac{1}{2} p_{n-1}^{k}
$$


When posed in this manner, the drunkard's walk is an example of a Markov process; eq. (7) is known as the Chapman-Kolmogorov relation.) Rewriting, we obtain.

$$
\frac{\rho_{n}^{k+1}-\rho_{n}^{k}}{\tau}=\frac{l^{2}}{2 \tau} \frac{\rho_{n+1}^{k}-2 \rho_{n}^{k}+\rho_{n-1}^{k}}{l^{2}}
$$

Taking the limit as $l$ and $\tau$ go to zero (but in a manner whereby $l^{2} / 2 \tau$ remains fixed) and noting that, from a first and second order Taylor expansion,

$$
\begin{aligned}
& \frac{\partial \rho}{\partial t}(x=n l, t=k \tau)=\lim _{\tau \rightarrow 0} \frac{\rho_{n}^{k+1}-\rho_{n}^{k}}{\tau}, \\
& \frac{\partial^{2} \rho(x=n l, t=k \tau)}{\partial x^{2}}=\lim _{l \rightarrow 0} \frac{\rho_{n+1}^{k}-2 \rho_{n}^{k}+\rho_{n}^{k}-1}{l^{2}}
\end{aligned}
$$

(where we have explicitly made the transition from a discrate to a continuous system), we find eq. (8) becomes the diffusion equation

$$
\frac{\partial \rho}{\partial t}=D \frac{\partial^{2} \rho}{\partial x^{2}}
$$

where the diffusion coefficient $D$ satisfies the relation

$$
D=\frac{1}{2} \frac{\ell^{2}}{\tau}
$$

Generally, $l$ and $\tau$ are called the collision mean free path and the collision time, respectively. The mathemacical 
Iimiting process just described can be performed rigorously. The essence of this demonstration is that there exists a precise method for describing random, stochastic processes via partial differential equations, a method known as potential theory. In practice, potential theory affords a much simpler and more reliable means of determining the behavior of a given population (whether it be composed of muskrats, drunkards or, we claim, extraterrestrials) than the associated random process (which, otherwise is only accessible to Monte Carlo methods).

Before returning to the specific problem of matching the dispersal of muskrats, let us explore some properties of the diffusion equation. As a generalization of (10), $:$ the diffusion equation can be witten

$$
\left.\frac{\partial O}{\partial t} \underset{\sim}{x}, t\right)=\nabla \cdot\{D(\underset{\sim}{x}, t, 0) \underset{\sim}{\nabla} \rho(\underset{v}{x}, t)\}
$$

where $p(x, t)$ now describes the population density. Here, the dimensionality of the process is increased in order to best represent the problem at hand (typicaliy, terrestrial problems are tro-dimensional). Tere $D$, the diffusion coefficient, need not be constant but, by this formulation, 
the conservation of the total population $\int o(\underset{\sim}{x}, t) d \underset{\sim}{x}$ is assured. (In such conservative cases, it is common practice to employ the terms population density and probability density interchangeably, although these quantities are of course not equal. The ratio of the first to the second is fixed, and the proportionality constant is the total population.) By allowing the diffusion coefricient to vary, we can, for example, represent the influence posed by geographical or environmental factors. When muskrats face a mountain range, their advance is slowed. In the present model, it follows that they will travel over a shorter distance $l$ in a time $\tau$ than would be the case for a flat plain; the diffusion coefficient drops correspondingly. As a result, the flow of emigration observed does not follow straight lines but describes an extremum path of least resistance. (Some sources tend to discount the utility of employing diffusive terms in population dynamics. They funish the erroneous counterexample that Minnesota would have been colonized by Europeans long before California, if the diffusion equation were employed. However, they overlook the point that D should be decreased to represent the difficulties faced in overland travel by settlers coming to Minnesota and increased. to describe the relative ease of ocean travel to California.) We see later how a population-dependent diffusion coefficient can be employed to 
describe social and territorial influences on emigration. For the sake of simplicity in our later discussion of interscellar colonization, we assume that space is homogeneous over a suitably large scale so that $D$ will have no spatial. variation. 'We shall, however, permit' the diffusion" coefficient to be population density dependent.

Since we will later be concerned with the problem of assessing the velocity of the colonization front, it is important to point out that the velocity associated with the diffusion process described by eq..(10) is not welldefined. For example, if we define $\left\langle x^{2}\right\rangle$, using the probability density $\rho(x, t)$ as

$$
\left\langle x^{2}\right\rangle=\int_{-\infty}^{\infty} x^{2} \rho(x, t) d x
$$

and integrate eq. (10) by parts twice, we find

$$
\frac{d}{d t}\left\langle x^{2}\right\rangle=2 D
$$

Thus, the quantity $\frac{d}{d t}\left\langle x^{2}\right\rangle^{\frac{1}{2}}$, which might be considered representative of the velocity, varies as $t^{-\frac{1}{2}}$. Einstein. (1905), in his classic microscopic treatment of. Brownian motion, showed that this anomaly only arose when timescales shorter than the collision time are considered. He demonstrated rigorously that the correct velocity at small times is $l / \tau$. Another way of characterizing the velocity $\forall$ is 
to compare the continuity equation (where $\underset{\sim}{\mathrm{j}}=\rho \underset{\sim}{\mathrm{V}}$ is the. f.1ux)

$$
\frac{\partial O}{\partial t}+\underset{\sim}{\nabla} \underset{\sim}{j}=0
$$

with (12); i.e., we make the association (known as Fick's law) that

$$
\underset{\sim}{j}=-D \underset{\sim}{\nabla} \rho
$$

or, alternatively,

$$
\underset{\sim}{V}=-D \rho^{-1} \underset{\sim}{\nabla} \rho
$$

Thus, the velocity associated with a diffusion coefficient independent of population density varies inversely as the density e-folding length but has no dependence on the density itself. In both of these descriptions, each measure of velocity possesses certain undesirable qualities which can be attributed to the fact that the solution to the diffusion equation (10) is spatially unconfined, because. the Green's function

$$
G(x, t)=(4 \pi D t)^{-\frac{1}{2}} \exp \left[-x^{2} / 4 D t\right]
$$

causes any initial configuration to be propagated and redistributed instantaneously over all space. We later 
see how nonlinear diffusion can overcome this velocity anomaly.

In practical situations, the diffusion coefficient is determined using Fick's law, i.e., as the quotient of the flux and the density gradient. In theoretical contexts, the diffusion coefficient must sometimes be determined-using other considerations. For example, discretizing (10) änd using length and time scales $\Delta x$ and $\Delta t$, respectively, we find

$$
\rho_{\mathfrak{n}}^{k+1}=D \frac{\Delta t}{\Delta x^{2}}\left(\rho_{\mathfrak{n}+1}^{k}+\rho_{\mathfrak{n}}^{k}-1\right)+\rho_{\mathfrak{n}}^{k}\left(1-\frac{2 D \Delta t}{\Delta x^{2}}\right)
$$

Thus, the effective probability $P$ of leaving site $n$ for either site $n-1$ or $n+1$ in a. time $\Delta t$ is given by

$$
P=2 D \Delta t / \Delta x^{2}
$$

Alternatively, the diffusion coefficient in m dimensions may be determined using the probability $P$, viz.

$$
D=P \Delta x^{2} / 2 m \Delta t .
$$

Despite our misgivings about the linear diffusion equation, it has proven remarkably successful in describing 
the dispersal of muskrats. Skellam (1951), citing earlier work, shows how well this model depicts the spread of Ondatra zibethica $\underline{L}$. since its introduction in central Europe in 1905. But muskrats are not extraterrestrials and the model must be adapted to include social influences on emigration patterns as well as the effects of population growth and saturation. (Skellam's treatment corrects for population growth, but only a posteriori). The possibility of an advanced civilization which practices zero population growth must be explored. Moreover, all this must be done in the context of estimating the velocity of an expanding population front.

\section{Density-Dependent Diffusion}

Consider, now, the case of a diffusion coefficient proportional to some power of the population density,

$$
D(\rho)=D_{\grave{o}}\left(\rho / \rho_{0}\right)^{N}
$$

For $N>0$, this generalization of the diffusion equation (12) has several conceptual advantages. From (17), we obtain a velocity that now has a population density dependence. From the probability expression (20) for the drunkard's walk, the likelihood that a drunkard will leave 
for another place now depends on how many other drunkards are converging on his lamppost. This is precisely the feature we desire: the probability of mounting a colonization venture is a rapidly increasing function of density, even if emigration cannot relieve the population pressure. [A more pronounced density dependence would arise by ușing an exponential dependence on the density. Crank (1956) investigated the problem numerically in certain isolated cases. The analytic properties of the solution, however, are not wel1-understood. For our purposes, the power law in (22) will suffice in describing a representative cross-section of density dependences.]

For the moment, we continue to neglect the homogeneous processes of growth and saturation. This might not be an unreasonable assumption in describing an advanced civilization. It seems likely that such a society would have undergone a long period of zero population growth while it developed the technological capability for interstellar flight. Otherwise, exponential growth would have diverted all available resources to the task of feeding a burgeoning population. ZPG must be an essential ethic of any society which is able to colonize other planetary systems. 
In the spherically symmetric three dimensional case, the population-dependent diffusion equation becomes

$$
\frac{\partial \rho}{\partial t}=\frac{1}{r^{2}} \cdot \frac{\partial}{\partial r}\left\{r^{2} \quad D_{\circ}\left(\frac{\rho}{\rho_{0}}\right)^{N} \frac{\partial \rho}{\partial r}\right\} \quad \cdot=
$$

In this form, the equation describes the "porous medium problem" and is frequently encountered in hydrology and soil science. [In the latter application, one considers the flow of a fluid through a porous medium. The mass of the fluid is conserved and it is assumed to obey a polytropic equation of state. Instead of the Euler force equation, the fluid is said to obey Darcy's law which provides for a velocity that is proportional to the pressure gradient. Muskat (1937) provides a definitive treatment of the problem. Philip (1970) offers an historical review of the subject including a treatment of selected topics in the theory of porous media.]

A formally identical equation is encountered in the physics of high-temperature gases, the "thermal wave" problem:

$$
\frac{\partial T}{\partial t}=\frac{I}{r^{2}} \frac{\partial}{\partial r}\left\{r^{2} \text { a } T^{N} \frac{\partial T}{\partial r}\right\}
$$


Here, $T$ is the temperature and the diffusion coefficient is temperature-dependent as would be the case in, for example, an ionized gas. Such a circumstance arises immediately after a high yield nuclear explosion, when temiperatures are sufficiently great that the velocity associated with radiative energy transport far exceeds the sound velocity. Since diffusion is now much more efficient at higher temperatures, it quickly acts to equilibrate the temperature distribution of the radiatively-heated gas. There is a thin boundary layer separating the hot gas from the ambient atmosphere that has not yet been heated by the radiation. In this boundary layer, called the "thermal wave front," the temperature undergoes a precipitous decline. Consequently, the thermal wave front is slow to conduct heat and the net temperature distribution changes slowly.

The overall result of these different effects is that the temperature distribution preserves a constant shape. Qualitatively, the temperature is relatively uniform. from the origin to the thermal wave front where it declines rapidly to zero. Quantitatively, the temperature (expressed in units of the 
temperature measured at the origin) is a function only of the radius (expressed as a fraction of the thermal wave front radius). To illustrate this, consider the one-dimerisional analogue of (24), .

$$
\frac{\partial T}{\partial t}=\frac{\partial}{\partial x}\left\{a T^{\mathbb{N}} \frac{\partial T}{\partial x}\right\}
$$

From this equation, we see that the quantity

$$
Q=\int_{-\infty}^{\infty} T(x, t) d x
$$

is conserved ( $Q$ is proportional to the total thermal

energy). There is only one dimensionless combination of the coordinate $x$ and the time $t$ that can be ob̈tained : in terms of $a$ and 0 using (25) and (26):

$$
\xi=\frac{x}{\left(a Q^{\mathbb{N}} t\right)^{1 /(N+2)}}
$$

The quantity $\left(Q^{2} / a t\right)^{1 /(N+2)}$ has the dimensions of temperature and a solution to (25) which preserves its shape is

$$
T(x, t)=\left(\frac{Q^{2}}{a t}\right)^{1 /(N+2)} f(\xi) \quad
$$

The solution for $f(\xi)$ (see Zel'dovich and Raizer, 1967, for details) is 
$f(\xi)= \begin{cases}{\left[\frac{\mathbb{N}}{2(\mathbb{N}+2)} \xi_{0}^{2}\right]^{1 / \mathbb{N}}\left[1-\left(\frac{\xi}{\xi_{0}}\right)^{2}\right]^{1 / N-},} & |\xi|<\xi_{0} \\ 0, & |\xi|>\xi_{0}\end{cases}$

where

$$
\xi_{0}=\left[\frac{(N+2)^{1+N} 2^{1-N}}{N \pi^{N / 2}}\right]^{1 /(N+2)}\left[\frac{\Gamma\left(\frac{1}{2}+1 / N\right)}{\Gamma(1 / N)}\right]^{N /(N+2)}
$$

For the case $N=0$ [i.e., eq. (10) taking $D \equiv a$,

$$
f(\xi)=(4 \pi)^{-\frac{1}{2}} e^{-\xi^{2} / 4}
$$

The normalization employed provides

$$
\int_{-\infty}^{\infty} f(\xi) d \xi=1
$$

and, for $\mathbb{N}>0$, the position of the thermal wave front, using (27), is just

$$
\mathrm{x}_{f}= \pm \xi_{0}\left(a Q^{\mathbb{N}} t\right)^{I /(N+2)}
$$


In Figure $1, f(\xi)$ is shown for $N=0,1$ and 2 . From eqs. (29) and (31), we see that:

a) if $N=0$, the distribution is unconfined;

b) if $0<\mathbb{N}<1$, the distribution has a finite cut-off where the temperature gradient vanishes;

c) if $\mathrm{N}=1$, the temperature distribution has a finite cut-off with a finite, non-vanishing. temperature gradient; and

d) if $\mathbb{N}>1$, the temperature distribution has a finite cut-off with an infinite temperature gradient.

The particular curves given in Figure 1 correspond to a Gaussian, a parabola and an ellipse, respectively. Apart from a differing normalization factor, the $f(\xi)$ profiles for spherical symmetry [eq. (24)] are unchanged. This solution to the porous medium or thermal wave problem was obtained independently by Barenblatt (1952) and by Pattle (1959). A thorough, physically motivated discussion of this problem is given in Zel'dovich and Raizer (1967). Knerr (1977) reviews some of the mathematical questions that arise in the one-dimensional version of this problem. 
As we have already noted, the shape of the temperature distribution is preserved in this solution. Such a solution is called "self-similar." IIn the Russian literature, solutions of this type are called "automodelled" solutions. Courant and Friedrichs (1948) were the first to consider such shape-preserving solutions in application to gas dynamic shocks; they employed the term "progressive waves."I Generally speaking, similarity solutions are characcerized by an equation of the type

$$
r_{f}=A t^{\alpha}
$$

where $I_{f}$ describes the radius of the phenomenon being studied (e.g., a shock front or a thermal wave), and $A$ and $a$ are constants.

There are two types of self-sinilar solutions. In the first, $a$ is obtained by dimensional analysis and $A$ is selected in order to satisfy a physical conservation law. The thermal wave problem is a case in point and eqs: (30) and 
(33) display the relevant features of the scaling. $2 /$

A second type of self-similar problem is characterized when $\alpha$ is selected to insure the existence of a mathematical solution. Since $\alpha$ is not known from dimensional considerations, the self-similar solution is usually obtained by exhaustive numerical tests. The standard example of such a problem is the implosion physics studied by Guderley (1942). In his case, $\alpha<0$ and trial values of $\alpha$ were varied (while numerically integrating the fluid dynamics equations) until a value of $\alpha$ was found that satisfied his boundary conditions. A more pertinent example of Type II self-similar solutions is that asso- . ciated with the semilinear diffusion equation in one dimension. (This equation is often used

2/ Another example of such so-called Type I selfsimilar solutions is that obtained for a strong isotropic blast wave, as when the thermal wave described earlier cools sufficiently for gas dynamic energy transport to be more efficient than diffusive dissipation. This solution was first obtained by Sedov (1959) in an application to supernovae. He argued that the shocked gas would lose its memory of its "initial" conditions due to convection and would approach a solution characterized only by the total energy $E_{0}$ of the original blast and the mass densicy $\rho_{0}$ of the unperturbed gas. In that case,

$$
r_{f}=\xi_{f}\left(E_{0} / o_{0}\right)^{1 / 5} t^{2 / 5}
$$

describes the radius of the shock, where $\xi_{f}$ is a dimensionless number of order unity selected to assurre that the blast energy equals the total internal and kinetic energy of the shocked gas. It is not without some irony that the methods for trearing explosive processes, such as thermal or blast waves, and, in particular, nuclear explosions, are valuable in studying population dynamics. 
in population dynamics modeling; we examine it in detail. shortly.) In that case, the solution for the density $\rho(x, t)$ can be expressed as a "travelling wave" $o(x-v t)$ where $v$ is the velocity of the travelling wave ("uniform propagation regime" in the Russian literature). If we take $x=\tilde{x}$ 名 $x$, and $t \tilde{t}=\tilde{t}$ \& $\tau$, then the solution can be witten as $\rho(\xi)$ where

$$
\xi=\chi / \tau^{\tilde{\mathrm{V}}}
$$

Comparing this with (34), we find that

$$
x_{\tilde{I}}=\xi_{f} \tau^{\tilde{v}}
$$

describes the position of the travelling wave front, where $\xi_{f}$ is a constant and $\tilde{v}$ has the same role as $a:$

$\because$ Although self-similar solutions are special solutions characterized by a dependence on a single. dimensionless variable, $\xi$, they appear to describe the "intermediate-asymptotic" behavior of a much broader class of initial, boundary and mired problems. In practice (for example, in the case of the thermal wave), the solution often loses its memory of the initial and boundary conditions. Then, the solution evolves into one characrerized 


\section{CATBMAL PAEE IS OS POOR QUALITY}

only by, e.g., conserved quantities (such as 0 ) and parameters that specify the problem (such as a). This property of self-similar equations has been rigorously demonstrated only for the linear diffusion equation (10): and the semilinear diffusion equation. Numerical and experimental results (where available) display the convergence of solutions to self-similar form under a broad array of initial conditions. Thus, self-similarity is not of interest simply because it facilitates the calculations, but also because it describes a seeming!y genuine asymptotic feature of the solution (see Barenblatt and Zel'dovich, 1972).

To recapitulate, we observe that a populationdependent diffusion coefficient overcomes many of the problems inherent in the conventional diffusion equation. From the self-similar solution, we observe that the distribution is confinied. Moreover, the velocity of advance of the. wavefront is no longer anomalous but can be calculated directly from eq. (33), for one dimension, or (for $N>0$ ) from

$$
r_{f}=\xi_{1}\left(a Q^{\mathbb{N}} t\right)^{\frac{1}{3 \mathbb{N}+2}}
$$


where

$$
\xi_{1}=\left[\frac{3 N+2}{2^{N-1} N \pi^{N}}\right]^{\frac{1}{3 N+2}}\left[\frac{\Gamma\left(\frac{5}{2}+\frac{1}{N}\right)}{\Gamma\left(1+\frac{1}{N}\right) \Gamma\left(\frac{3}{2}\right)}\right]^{\frac{N}{3 N+2}}
$$

in three dimensions (Zel'dovich and Raizer, 1967). The yelocity monotionically decreases as a function of time. (This comes about since the central density decreases causing the diffusion rate to diminish accordingly.) Although this model ignores homogeneous processes, it is a useful description of situations where the overall population is conserved (ZPG) and provides useful insight into models that combine both homogeneous and heterogeneous processes.

4. Diffusion and Growth

As a first step toward simulating the combined effects of homogeneous and heterogeneous processes, consider the equation

$$
\frac{\partial \rho}{\partial t}=\alpha \rho+a \frac{I}{r^{2}} \frac{\partial}{\partial r}\left[r^{2} \rho^{\mathbb{N}} \frac{\partial \rho}{\partial r}\right]
$$

This is similar to an approach first proposed by Gurney and Nisbet (1975). By employing a population-dependent diffusion coefficient, they suggest that one can describe 
one non-random cause of dispersal, the "directed-motion" associated with territorial drives. (They confined their attention to the case $N=1$, although they did allow for an additive third term describing random motion; this they called the biased random motion model.) Amplifying on this theme, Gurtin and MacCarey (1977) proposed a method of solution for the one-dimensional analogue of (39),

$$
\frac{\partial \rho}{\partial t}=\mu \rho+\frac{\partial^{2}}{\partial x^{2}} k \rho^{\alpha}
$$

After making the substitutions

$$
\begin{gathered}
\tilde{\rho}=e^{-\mu t} \rho, . \tau=[(\alpha-1) \mu]^{-1}\{\exp [(\alpha-1) \mu t]-1\}: \\
x^{\prime}=x(k \alpha)^{-\frac{1}{2}}, \quad N=\alpha-1,
\end{gathered}
$$

we obtain

$$
\frac{\partial}{\partial \tau} \tilde{\rho}=\frac{\partial}{\partial x^{T}}\left\{\tilde{\rho}^{N} \frac{\partial \tilde{\rho}}{\partial x^{\top}}\right\}
$$

We have already discussed the solution to the latter equation [via eqs. (25) through (30)]. However, because of the scalings employed in (41), we observe that $\rho(x=0, t)$ must ultimately increase exponentially with time. To eliminate this anomaly, we must prevent the population from growing above the local carrying capacity. 


\section{The Semilinear Diffusion Equation}

We wish to describe the behavior of an organism with advantageous genes which undergoes random dispersal while increasing in population locally according to the Pearl-Verhulst logistic law. In one dimension, this can be written

$$
\frac{\partial \rho}{\partial t}=\gamma \rho\left(1-\rho / \rho_{s}\right)+\frac{\partial}{\partial x}\left\{D \frac{\partial \rho}{\partial x}\right\}
$$

where $\gamma$ is the local growth rate, $\rho_{s}$ is the local carrying capacity (or population-density saturation level) of the environment and $D$ is the local diffusion coefficient (assumed here to be population-independent). Further, it is assumed that $\gamma, \rho_{S}$ and $D$ are constant and do not vary spatially or temporally. Making the transformations

$$
\begin{aligned}
& \rho^{\prime}=\rho / \rho_{s}, \quad t^{\prime}=\gamma t \\
& x^{\prime}=x(D / \gamma)^{-\frac{1}{2}},
\end{aligned}
$$

eq. (43) becomes

$$
\frac{\partial \rho}{\partial t}=\rho(1-\rho)+\frac{\partial^{2}}{\partial x^{2}} \rho
$$

(where, for convenience, the primes have been dropped). 
Fisher (1937) and Kolmogoroff, Petrovsky and Piscounoff (1937) independently proposed that eq. (45) has a right-going travelling wave solution. [The relationship of the travelling wave to self-similar... solutions was reviewed in the discussion leading to eqs. (35) and (36).] Assuming, then, that $p$ depends only on $x$-vt in (43), we first transform the velocity $v$ into

$$
\tilde{\mathrm{v}}=\mathrm{v}\left(\mathrm{D}_{\gamma}\right)^{-\frac{1}{2}}
$$

where $\tilde{\mathrm{V}}$ is a dimensionless.quantity, and, employing (44) and (45), obtain the ordinary differential equation

$$
-\tilde{v} \frac{d \rho}{d x}=\rho(I-\rho)+\frac{d^{2}}{d x^{2}} \rho
$$

We wish now to find the range of $\tilde{\mathrm{v}}$ for which eq. (47) possesses a solution. (Recall from our earlier discussion that Type II similarity solutions are characterized by a dimensionless constant, in this instance $\tilde{v}$, whose value is chosen in order to insure the existence of a mathematical solution.) Since we assumed a right-going travelling wave, we should expect the density $\rho$ to decrease monotonically in the direction of increasing $x$. Further, we assume that

$$
\begin{array}{ll}
\lim _{x \rightarrow+\infty} & \rho(x)=0 \\
\lim _{x \rightarrow-\infty} & \rho(x)=1
\end{array}
$$


i.e., that the density vanishes in the region unaffected by the travelling wave, while the source of the organism (infinitely far off to the left) is supported at the carrying capacity. Since $\rho$ is assumed monotonic in $x$, we can replace the derivatives in (47) according to

$$
\frac{d}{d x}=-q \frac{d}{d p}, \quad q \equiv-\frac{d p}{d x}
$$

( $q$ is defined so that it is necessarily positive-valued). Then, (47) becomes

$$
\tilde{\mathrm{v}} q=\rho(I-\rho)+\dot{q} \frac{d}{d \rho} q . \quad .
$$

Near the origin (i.e., $\rho=0$ ), this equation has the asymptotic representation

$$
\tilde{v} q \simeq \rho+q \frac{d q}{d o}
$$

Since we expect that $q$ vanishes when $\rho=0$, consider a solution of the form

$$
q=b \rho^{n}
$$

where $b$ and $n$ are real-valued positive constants. Substituting into (5I), we find that $n=1$ and

$$
\tilde{\mathrm{v}} \mathrm{b}=1+\mathrm{b}^{2}
$$


Solving for $\mathrm{b}$ in terms of $\tilde{\mathrm{v}}$, we obtain

$$
\mathrm{b}=\left[\tilde{\mathrm{v}}+\cdot\left(\tilde{\mathrm{v}}^{2}-4\right)^{\frac{3}{2}}\right] / 2
$$

Therefore, in order to guarantee that $b$ be real-valued, the discriminant must be positive and

$$
\tilde{\mathrm{v}} \geq 2
$$

(The case $\tilde{\mathrm{v}} \leq-2$ corresponds to a left-going travelling wave.)

Although Fisher and Kolmogoroff et al. obtained this result, Kolmogoroff et al. went on to show that the. solution to the partial differential equation (45), initialized as a step function, converges to a right-going travelling wave with the minimal velocity $\tilde{v}=2$. Although a continuous spectrum of propagation velocities $\tilde{v} \geq 2$ is in principle possible, only the solution corresponding to the extreme point of the spectrum $(\tilde{v}=2)$ can be an asymptotic solution to the partial differential equation (45). Therefore, the velocity of the front of advance of the advantageous genes, in Fisher.'s formulation of the problem, "converges" to $2(\mathrm{D} \gamma)^{\frac{1}{2}}$. 
Although this model from population genetics can. be made more general by employing a broader description of in situ growth processes than that provided by. the Pearl-Verhulst 1aw, : such models are characterized by random dispersal, i.e., a population-independent diffusion coefficient (see, for example, the review article by Hadeler, 1977). Characteristically, they have travelling wave solutions which, when normalized in a similar fashion, also give $\tilde{v}=2$. Thus, despite the presence of a linear diffusion term, the associated velocity of advance is welldefined [as contrasted with the absence of a self-consistent velocity for the linear diffusion model in the absence of population growth, ẹ. (10)].

There is, however, one feature of this model (apart from the population-independent diffusion coefficient) that is undesirable. From eq. (54), we see that $b=1$ when $\tilde{v}$ is minimal and, from (49) and (52), we find

$$
\rho(x)=e^{-x}
$$

when $\rho$ is small. Therefore, the population's travelling wave front is unconfined. [This again parallels the result 
obtained for the Iinear diffusion model (10).]

This model has been employed in describing the interaction of several species. For example, in a predator, denoted by $\rho_{1}$, and its prey, denoted by $\rho_{2}$, might be written

$$
\begin{aligned}
& \frac{\partial \rho_{1}}{\partial t}=-c_{1} \rho_{1}+c_{2} \rho_{1} \rho_{2} \\
& \frac{\partial \rho_{2}}{\partial t}=c_{3} \rho_{2}-c_{4} \rho_{1} \rho_{2}
\end{aligned}
$$

where $c_{1}, c_{2}, c_{3}$ and $c_{4}$ are positive constants. In the absence of prey, the number of predators will decay exponentially. On the other hand, in the absence of predators, the number of prey will grow exponentially. When both predators and prey are present, the growth rate of predators increases while that of the prey decreases. This formulation of the predator-prey relationship is generally associated with the names of Lotka and Volterra (see Davis, 1962, for a simple treatment of the mathematical theory), and describes accurately a number of wellobserved ecosystems. The solutions to eqs. (57) describe closed orbits in the $\rho_{1}^{-\rho_{2}}$ plane and are akin to the solution for a harmonic oscillator in that $\rho_{1}$ and $\rho_{2}$ will 
oscillate in a pattern' strictly determined by initial conditions. When eqs. (57) are augmented by linear diffusion terms $\mathrm{D} \partial^{2} \rho_{1} / \partial \mathrm{x}^{2}$ and $\mathrm{D} \partial^{2} \rho_{2} / \partial \mathrm{x}^{2}$, respectively, our -equations assume more of the character of the semilinear diffusion equation (43). An example of the predator-prey relationship is seen in herbivorous copepods (zooplankton) which feed on phytoplankton. In performing a linearized perturbation analysis for such marine planktonic communities, Steele (1974) noted that ali finite wavelength perturbations will be damped and the ecosystem will tend to spatial homogeneity. Nonlinearities, however, could conceivably destabilize the situation. Levin (1976) and McMurtrie (1978) have reviewed the multi-species population dynamics problem.

In the case just described, diffusion acts to stabilize the system (at least in a linearized representation). In other circumstances, diffusion can produce a travelling-wave similar, in certain respects, to that of the semilinear diffusion equation $\left(4 \dot{3}^{3}\right.$. This feature was 
employed by Noble (1974) $3 /$ in devising a model for the Bubonic Plague of 1347. His equations are

$$
\begin{aligned}
& \frac{\partial I}{\partial t}=K I S-\mu I+D \nabla^{2} I . \\
& \frac{\partial S}{\partial t}=-K I S+D \nabla^{2} S
\end{aligned}
$$

where $S$ and $I$ are the densities of the susceptible and the infected populations, $\mu$ is the disease's mortality rate, and $D$ is the diffusion coefficient. The coefficient $\mathrm{K}$ describes the rate at which the plague is transmitted locally. (There is, in fact, a third "species" implicitly included here, the density of individuals $B$ who have contracted the disease and either perished or recovered. The "mortality" rate $\mu$ describes the rate at which this third population density grows, $\partial B / \partial t=\mu B$. The total population

3/ Noble compared his model predictions with the information available on the Black Death (Langer, 1964). His results appear to match what is known about the geographic advance of the disease. In commenting on the applicability of his model to other epidemiological problems, he suggested that the spread of certain mass sociopsychological phenomena (such as new religions) might be sufficiently similar to that of plagues to be worth investigating by similar methods. It is possible that the spread of UFO belief systems might be described in this way. 
$\int(I+S+B) d^{2} X$ then remains constant.) Eq. (58) represents the rate of change of the number of infectives within a small area as the rate of transitions (KIS) from the susceptible population less the removal rate (due to mortality, $-\mu I$, and dispersal $\left.D \partial^{2} I / \partial x^{2}\right)$. Meanwhile, the rate of change of the susceptible population within a small area is a net loss due to the transition to the infected population (-KIS) and dispersal (D $\partial^{2} S / \partial x^{2}$ ). Noble performed a numerical integration of the one-dimensional analogue of eqs. (58). His simulation quickly evolved into a pair of travelling waves, one describing the infectives and the other, the susceptibles. The qualitative shape of the two population distributions is depicted in Figure 2. Thus, we see that semilinear diffusion acting in a multispecies problem can produce a set of travelling waves just as we observed in the single species case:

\section{Density-Devendent Diffusion with Growth and Saturation}

We have already described the need for a population dynamic model that combines local growth and saturation processes with spatial dispersal mechanisms that are population-density dependent. This model, in one dimension, 
may be expressed as

$$
\frac{\partial \rho}{\partial \tau}=\gamma \rho\left(1-\rho / \rho_{S}\right)+\frac{\partial}{\partial x} D\left(\frac{\rho}{\rho_{S}}\right)^{\mathbb{N}} \frac{\partial \rho}{\partial x}
$$

where the diffusion constant $D$ is scaled in order to provide the diffusion coefficient when $\rho=\rho_{S} \cdot 4 /$ From the transformations (44), we obtain an equation analogous to (45), namely

$$
\frac{\partial \rho}{\partial t}=\rho(1-\rho)+\frac{\partial}{\partial x} \rho \mathbb{N} \frac{\partial \rho}{\partial x}
$$

We have investigated the properties of the solution to eq. (60) for various $N$ by numerical means. The equation was integrated using a conventional four-point. explicit integration scheme (Richtmeyer and Morton, 1967).

4/ Density-dependent diffusion does not make emigration a response to population pressure alone: Since the flux of the population is given by $-D\left(o / \rho_{S}\right){ }^{N o} / \partial x$, the density gradient plays an all-important role.- Thus, for example, there is little net movement of a population that is approximately uniform in density, even if the density is nearly saturated. 
The density distribution, following Kolmogoroff et al. (1937), was initialized as a step function

$$
\rho(x, t=0)=\left\{\begin{array}{ll}
1 & x<0 \\
0 & x>0 .
\end{array} .\right.
$$

Since we were also interested in the spherically symetric case, we numerically integrated

$$
\frac{\partial Q}{\partial \tau}=0(I-0)+\frac{I}{I^{2}} \frac{\partial}{\partial r}\left\{r^{2} 0^{N} \cdot \frac{\partial O}{\partial r}\right\}
$$

using a stable differencing scheme developed by Eisen (1967) in applications to the spherically symetric diffusion equation. The initial conditions employed were

$$
p(r, t=0)= \begin{cases}I & I<r_{f} \\ 0 & I>r_{f}\end{cases}
$$

wherem $I_{\text {f }}$ is some arbitrary radius. As a test of the integration methods, the case of $N=0$ was included. In both geometries, for $\mathbb{N}=0, I$ and 2 , the numerical solution evolved into a travelling wave. (The asymptotic correspondence between the one dimensional and spherically symetric cases is nor surprising. The only difierence between their respective equations is $(2 / r) p^{N} \partial \rho / \partial r$. Assuming that the solution tends to the form $\rho(r-v t)$, this 
term makes a contribution of order $t^{-1}$, which at large times is negligible.) Since, for $N=0$, the numerically evolved travelling wave has a velocity very near 2, we consider the asymptotic convergence of the solutions (for $N=1$ and 2) to travelling waves to be significant. Figure 3 illustrates the density profile for these three cases. In order to facilitate a comparison between the wave fronts, the density profiles are constructed so that density is one-half at the origin.

Several other interesting feacures of the solution emerge from the computer analysis. First, the velocity $\tilde{v}$ of the travelling wave $[$ eq. (46)] decreases with increasing $\mathrm{N}$. The values obtained for $\mathbb{N}=1$ and 2 were approximately 0.70 and 0.45 respectively. (Higher values of $\mathrm{N}$ were also investigared. Fluctuations in $\tilde{\mathrm{v}}$ made these cases difficult to estimate, but the decline of $\tilde{v}$ with increasing $N$ was unmiscakable.) Secondly, the densicy profile near the point of vanishing density resembled the self-similar thermal wave profiles for $N=1$ and 2 which appear in Fig. 2 . 
Consider, therefore, the problem of finding a traveling wave solution to (60). Employing the methods used earlier in our discussion of the semilinear diffusion equation, we write the equation for $o(x)$ as.

$$
-\tilde{v} \frac{d \rho}{d x}=\rho(1-\rho)+\frac{d}{d x}\left\{\rho \frac{d \rho}{d x}\right\} \text {. }
$$

Then, making the same assumptions here as were employed in obtaining (50), we have

$$
\tilde{\mathrm{v}} q=\rho(1-\rho)+\dot{q} \frac{d}{d \rho}\left(\rho^{\mathbb{N}} q\right)
$$

As before, we obtain an approximate solution as a power law. [eq. (52)], namely

$$
q \simeq\left\{\begin{array}{ll}
\tilde{v} \rho^{1-N}, & \dot{N}>0 \\
\frac{1}{2}\left[\tilde{v}+\left(\tilde{v}^{2}-4\right)\right] \rho & N=0
\end{array} \cdot\right.
$$

Near $\rho=1$, the solution is given approximately by

$$
q \approx \frac{1}{2}\left[\left(\tilde{v}^{2}+4\right)^{\frac{1}{2}}-\tilde{v}\right](1-0)
$$

for all N. Integrating (67), we see that the density exponentially decays to unity as we move inside the travelling wave. Near the front of the travelling wave, the density (for the $\mathbb{N}=0$ case) decays exponentially. 
However, for $\mathrm{N}$ nonzero, the solution is of the form

$$
\rho(x) \simeq\left[N \tilde{v}\left(x_{c}-x\right)\right] 1 / N
$$

where $\mathrm{x}_{c}$ denotes a cut-off point to the right of which the density is identically zero. This behavior is èssentially the same as we observed in the thermal wave problem for $N>0$ [see eq. (29) and subsequent discussion] and explains the striking similarity observed in comparing the $\mathbb{N}=1$ and 2 curves in Figs. 1 and 2 (the thermal wave and travelling wave fronts, respectively). What has occurred is that, for $N>0$, the growth and saturation term in eq. (64) has no influence on the shape of the density profile near the cut-off point and a solution reminiscent of the self-similar thermal wave problem emerges. The growth and saturation terms, however, have a controlling influence on the determination of $\tilde{\mathrm{v}}$.

Upon finding this asymptotic behavior, we noted that (67) was an exact solution to (65) for $N=1$ if we set $\tilde{v}=2^{-\frac{1}{2}}$. A straightforward integration then gives

$$
\rho(x)= \begin{cases}1-\exp \left[2^{-\frac{1}{2}}\left(x-x_{c}\right)\right], & x<x_{c} \\ 0, & x>x_{c}\end{cases}
$$


This profile corresponds, within the numerical limitations posed by the integrarion scheme, to the computed solution and the predicted travelling wave velocity of $2^{-\frac{y}{2}}$ corresponds well with. the numerical result of 0.70 . Although we are not at present able to demonstrate the stability of this solution (in the sense of Kolmegoroff et a..), the convergence observed in the numerical solution to the profile. given by eq. (69). is a relacively convincing demonstration of some form of asymptotic stability. However, unlike the case of the Fisher-Kolmogoroff. problem, we have explicitly obcainad a closed form expression for the population density profile.

5/ Lax (1969), in presenting the ninth John von Neumann Lecture of the Society for Industrial and Applied Mathematics remarked on how fascinated von Neumann had become by the possibility that patterns disclosed by numerical calculations inight reveal entirely unsuspected properties of solutions of nonlinear differential equations. A case in point is the Kortweg-deVries equation which. describes long waves over water and some wave phenomena in plasma physics. In his lecture, Lax went on to describe how Kruskal and Zabusky discovered the existence of certain solitary wave solutions after studying motion pictures of the computations. In our case, the computed solutions to eq. (59) pointed out the existence of an asymptotic travelling wave solution and, in one instance, led to a closed-form analytic expression for the profile and its associated traveling wave velocity. 


\section{Summary of Mathematical Approaches}

We have reviewed the advantages (and limitations) of potential theory in describing population dynamic processes. We have observed that dispersal is essentially a diffusion process whereas population growth (if it takes place) is restricted by the carrying capacity of the environment. A density-dependent diffusion coefficient was shown to be capable of describing the desirability of emigration (all other factors being equal). Essentially, two basic models emerged. One model described a condition of zero population growth. The properties of its solution may be obtained from the porous medium or thermal wave problems. The second model also included local growth and saturation. A numerical study of its properties disclosed the existence of an asymptotic travelling wave solution.

Two features emerged from our population dynamic modelling. First, the velocity of the colonization wave front can be estimated by dimensional analysis using the physical and other parameters that influence the evolution of the civilization. The parameters that contribute to this problem include the growth rate $\gamma$ and the diffusion coefficient $D$. The latter, in turn, is related by eq. (21) to 
the effective probability $P$ that an individual member of a civilization will embark on such a venture, the mean distance $\Delta x$ between habitable solar systems and the effective time span $\Delta t$ between successive colonization ventures (to be discussed below). Second, the dimensionless multiplying coefficient that appears in the expression for the velocity ( $\xi$ or $\tilde{v}$ for the zero population growth and travelling wave solutions, respectively) is of order unity for all models and the explicit dependence of the velocity on the physical parameters is characterized by power laws with fractional exponents. This manifests itself in velocities that are remarkably and encouragingly insensitive to the particular model and parameters employed. In the next section, we see how both models, applied to interstellar colonization, yield a much lower colonization wave velocity than had previously been predicted.

Before continuing, we should point out why our models provide results that contrast so strongly with those of Jones (19.76). We recall that, in his model, all saturated solar systems were sources of colonists. In all of our 
diffusion models that include population growth and saturation, the flux of colonists goes as $\rho^{\mathbb{N}} \partial \rho / \partial r$ (where we now employ spherical symmetry). From our computed solutions, the flux is significant only in a thin layer at the population wave front. In both Jones' and our models, colonists are the source of population growth (all growth has vanished from the saturated interior region). In our case, however, colonists are produced only near the surface of the sphere enclosing an interstellar civilization. We stress that this qualitative understanding of the difference between our models and that due to Jones evolved only after exploring the methods and assumptions implicit in current population dynamic models. It was only after performing an extensive analytic and numerical investigation of the equations of population dynamics that we were able to distinguish the two most prominent features of population dynamic models: expansion due to directed diffusion from the colonization front and a velocity of expansion that is, at the same time, characterized by but relatively insensitive to the physical and sociological parametiers involved, 
In each case, the rate of populacion increase du/dt is proportional to the number of colonists $\theta$ present, i.e.

$$
\mathrm{d} v / \mathrm{dt}=\mathrm{A \theta}
$$

In our case, $\theta$ is proportional to the

surface area of the saturated sphere containing the expanding interstellar civilization. Since $\nu$ varies as the radius of the sphere, $I_{f}$, to the third.power, our model predicts

$$
\theta=B v^{2 / 3} \quad ;
$$

Jones' model predicts

$$
\theta=B^{\prime} v
$$

Combining eqs. (70) and (7I) gives

$$
v=t^{3}
$$

Therefore, our model predicts that $I_{f}$ * $t$ and we have a. colonization sphere that is undergoing uniform expansion. On the other hand, Jones' model [eqs. (70) and (72)] yields

$$
v=\exp \left(A B^{\prime} t\right)
$$

Consequently, his model gives an exponentially growing colonizacion front velocity. The critical difference between Jones' model and ours is that our model supplies colonists only from the periphery of the civilization. 
Since we expect the core of the civilization to be saturated, almost all available resources would by definition be committed to the continued maintenance of the local ecosystem and few, if any, resources would be available for the construction of starships capable of transporting and supporting colonists across interstellar space. Interstellar spaceflight for a substantial community of colonists at vic. must for any civilization be enormously expensive in propulsion and shielding. At $\mathrm{v}<<\mathrm{c}$, the cost remains enormous because the long transit times require elaborate life-support systems. Contemporary colonies of $\sim 10^{4}$ persons in cislunar space (O'Neill, 1975, 1978) are estimated optimistically to cost $>>10^{11}$. Fven with generous allowances for future technological progress a larger colony designed to traverse parsecs safely might be argued to cost substantially more than the available resources of the planet Earth ( $\$ 10^{13}$ gross planetary product). It is not clear that for either velocity regime, colonization ventures to more distant targets would be correspondingly more expensive. Except for extremely advanced and long-lived technical societies, colonization is possible only to the nearest star systems. Thus colonization is initiated from the periphery of an empire for two reasons. First, the transit distances and times are shorter. Second, 
materials are not in such short supply and the launching of colonization ventures would not place as unbearable a strain on the outposts of the empire as on its central worlds.

\section{Results}

We now collect some relevant results of the preceding sections, apply numerical values, and address ourselves to the central question of whether the Earth should have been visited during geological time by extraterrestrial civilizations. 
The steady state number $\mathrm{N}$ of extant civilizations more advanced than our own in the Milky Way Galaxy can be written as

$$
\text { - } N=f I,
$$

where $L$ is the mean lifetime of galactic civilizations in years and $f$.is a factor which combines the rate of star formation, the fraction of stars with planetary systems, the number of ecologically suitable planets per such system, che probability of the origin of life on a given otherwise suitable planet, and the likelihood of the evolution of intelligence and technical civilization (for extensive discussions, see, for example, Shklovskii and Sagan, 1966; Sagan, 1973b). A conventional estimate, which is, however, no more than a semi-informed guess, is $f$ $v 10^{-1} \mathrm{yr}^{-1}$. However, in the case of colonization the probability of the emergence of intelligent life and technical civilization on a given world approach unity and this factor increases perhaps to $f$ ilo $\mathrm{yr}^{-1}$ (Shklovskii and Sagan, 1966, p. 45I). 
Assuming stars in the Milky Way Galaxy to have a mean separation of 1 parsec $(=3.26$ light years $)$, the mean distance between advanced civilizations is

$$
\Lambda \simeq\left(2.5 \times 10^{1 I / N}\right)^{1 / 3}=\left(2.5 \times 10^{11} / \mathrm{II}\right)^{1 / 3} \mathrm{pC} .
$$

We here assume 250 billion stars in the Galaxy. Henceforth, distances will be measured in parsecs and times in years. With a conventional value (oD. cit.) for independently arisen civilizations, of $\mathrm{N}=10^{6}, \Lambda=25 \mathrm{pc}=82 \mathrm{l} \cdot \mathrm{y}$. Eq. (76) is based on a spherical distribution of stars in the Galaxy; but the inaccuracies for distances larger than the thickness of the Galaxy ( 100 pc) is still small enough that we may neglect it in this problem.

For our density-dependent diffusion problem with growth and saturation, we have found that: the velocity $v$ of the colonization front can be represented by

$$
\nabla=\tilde{v} \quad(D \dot{y})^{1 / 2}
$$

where $\tilde{v}$ is a dimensionless constant of order unity; $D$ is a diffusion coeficiene that, when multiplied by the population density gradienc, yields the correct outward flux of population as it nears saturation, and $\gamma$ is the population growth race. For the human population on the 
Earth today, $\gamma \sim 10^{-2}$. In the cencuries prior to 1750 , $\gamma$ was $\sim 6 \times 10^{-4}$ (Coale, 1974). In the late Pleistocene $\gamma$ was much smaller still, perhaps $10^{-7}$ or $10^{-8}$.

We have found [eq. (21)] from finite differences in the standard m-dimensional diffusion equation that the probability of diffusing to another planet $\Delta x$ away in time $\Delta t$ is $P=2 m D \Delta t / \Delta x^{2}$. This is in fact the probability of the entire population emigrating to another solar system at a distance $\Delta x$ over an effective time $\Delta t$. This is in strict agreement with the behavior of a particle undergoing Brownian motion. Fowever, since the diffusion equation describes the behavior of a distribution of particles, it is conceptually useful to think of $P$ as the expectation value of the fraction of the population that will emigrate to another world at a distance $\Delta x$ in a time $\Delta t$. In the sense of an expectation value, then, we define the specific emigration rate $\Psi$ by

$$
\Psi=P / \Delta t=2 m D / \Delta x^{2}
$$

This quantity describes the fraction of the population that emigrates per unit time to the next solar system. Here, $\Delta t$ is the time interval between successive colonial ventures. If the transit time exceeds this time interval then we 
substitute the transit time for $\Delta t$. Conversely, the problem is paced by the time interval between successive colonizations for very fast transit times, so that even the extremely unlikely contingency of faster-than-light travel (through. multiply-connected space in the vicinity of black holes, say) does not alter this analysis significantly.

It is impossible to make a very reliable estimate of $\Psi$ for extraterrestrial civilizations. Very high values should be stupifyingly expensive. Very low values cannot provide even short-term relief of population pressure at the periphery of the civilization. In the third century B.C, the Chin emperor approved a proposal of Hst Fu to launch a colonization mission "with several thousand young men and maidens to go and look for the abodes of the immortals hidden in the Eastern Ocean." (Needham; 1971). The fleet of sailing. ships was never heard from again and the emperor complained bitterly about the cost. Over the following centuries a number of further expeditions to the Pacific Ocean to find an elixer of immortality were mustered, but none on the scale of the venture of Hsu Fu (op. cit.). All these expeditions may have perished; altematively some may have colonized Japan and have been the origin of the non-Ainu population 
there. At this time the total population of China was $\sim 3 \times 10^{7}$. The next major distant emigrations from East and Northeast Asia were the Mongol invasions and the exploration and trade by the Ming Navy in the Indian Ocean in the fifteenth century. These numbers give $\Psi \sim 10^{-7} \mathrm{yr}^{-1}$. During the eighteenth century European colonization of North America, $\Psi$ was $\sim 3 \times 10^{-4} \mathrm{yr}^{-1}$ (Potter, 1965). Proposals have been made recently for the launching into Earth orbit. of self-çontained closed ecological systems with $\sim 10^{4}$ inhabitants ( $\left.0^{\prime} \mathrm{Neil1}, 1975,1978\right)$. This represents $210^{-6}$ of the population of the Earth. A very difficult undertaking in the next few centurïes would be to launch one suich Späce City into interstellar space every century. This gives $\Psi \sim 10^{-8} \mathrm{yr}^{-1}$. We adopt $3 \times 10^{-4} \mathrm{yr}^{-1} \geq \Psi>10^{-8} \mathrm{yr}^{-1}$, with a bias to the smaller value for a younger interstellar civilization.

The existence of abundant extra-solar planetary systems seems very plausible but is still undemonstrated. Presumably a civilization wishing to colonize the Earth comes from an Earth-like world; although, since schemes have been proposed to terraform Venus and Mars -- that is, to convert them from rather different environments to ones 
which resemble the Farth (Sagan, 1960; Sagan, 1973c) -xenoforming the Earth into some rather different environment might not be beyond the capabilities of an interstellar spacefaring civilization. We note that such xenoforming will add to the waiting time for a colony to develop an independent colonizing capability. Simple theoretical models of the formation of planetary syscems from solar nebulae suggest that a wide variecy of planetary systems can be formed depending on, for example, the initial mass density distribution funcrion of the nebula; in some schemes terrestrial planets appear to be formed abundancly' (Isaacman and Sagan, 1975). In this case the distance between Earthlike planets is probably $\leq 3 \mathrm{pC}$ (see also Dole, 1964). Hart (1978, 1979) has argued that only an extremely unlike1y, set of circumstances has preserved the Earth's environment -particularly abundant liquid water -- from the opposing threats of a runaway greenhouse effect and a global ice age. These arguments. ignore plausible variations in some climatic parameters and neglect negative feedback loops in terrestrial climarology; we consider them unlikely. But if they are valid they increase $\Delta x$ possibly to 300 pc. Accordingly, we will allow $d \mathrm{r}^{2}$ to range from $3 \mathrm{pc^{2 }}$ to $10^{3} \mathrm{pc}^{2}$, with a prafierred value $0 \leqslant 10 \square c^{2}$. 
If we adopt $\mathrm{m}=3, \Delta \mathrm{x}^{2}=10 \mathrm{pc}^{2}$, and $\Psi=10^{-8} \mathrm{yr}^{-1}$ we find a typical value of the diffusion coefficient to be $\mathrm{D}=2 \times 10^{-8} \mathrm{pc}^{2} \mathrm{yr}^{-1}=5 \times 10^{21} \mathrm{~cm}^{2} \mathrm{sec}^{-1}$, an absolutely enormous diffusion coefficient by planetary atmospheres standards.

We are now ready to examine the apparent absence of extraterrestrial colonies on the Earth. Let $t_{\min }$ be the minimum time for the advanced technical civilization nearest us in space to reach us at a diffusion wavefront velocity $v$. Then $v t_{\min }=\Lambda$. If $t_{\min }>\mathrm{L}$, there should be no such colonies, as observed. Consequently, there is a critical lifetime for a spacefaring intersteilar civilization

$$
I_{c}=t_{\min }=\dot{\Lambda} / \mathrm{v}
$$

For the Earth to have been colonized, the colonial civilization must have a lifetime in excess of $\mathrm{L}_{\mathrm{c}}$.

Combining eqs. (76)-(79) we find

$L_{c}=3.3 \times 10^{7}(E / 0.1)^{-1 / 4}\left[\left(Y / 10^{-4}\right)\left(\Psi / 10^{-8}\right)\left(\Delta x^{2} / 10\right)\right]^{-3 / 8}$ yrs. (80) For comparison, we combine eqs. (23), (24), (26), (76) and (78) to obtain the critical lifetime for a thermal wave front 
with $\mathrm{N}=2$ and find

$I_{c}=1.3 \times 10^{10}(f / 0.1)^{-8 / 11}\left(\Delta x^{2} / 10\right)^{-12 / 11}\left(\Psi / 10^{-8}\right)^{-3 / 11}$ yxs.

This is the case of strictly observed zero population growth. Our free parameters, $f, Y, \Delta x^{2}$ and $\Psi$ have normalized standard values, already discussed, with distance measured in pc and time in years.

We have now obtained the critical

Iifetime $I_{c}$ for the cases $I_{c}<y^{-I}$. (zero population growth) and $I_{c}>\gamma^{-1}$ (nonlinear population diffusion with growth and saturation). From eq. (80), we see that $L_{c}$ increases as $\gamma$ decreases. When $\gamma$ is set equal to $I_{c}{ }^{-1}$ in $(80)$, we obtain a value for $I_{c}$ of $4.3 \times 10^{9}$ yrs when $f$, w and $\Delta x$ assume their nomalized standard values. Although (80) is not strictly valid in this case, this value for $L_{c}$ suggests, as we intuitively expect, a smooth transition between our two models, i.e., as $\gamma$ decreases, $I_{c}$ increases until it assumes the ZPG value.

We ses that for the colonization wavefrone of the nearast technical civilization to have reached the Earth, the lifetime of that civilization must exceed 30 million yrs. 
for nonlinear diffusion with growth and saturation. Such a civilization will have been intensely occupied in the colonization of more than 200,000 planetary systems before reaching the Earth, some 64 pc away. Many colonial empires, of vast extent by terrestrial standards, may still occupy only an insignificant volume of the Milky Way Galaxy, and not embrace the nearest independently arisen technical civilization whether it has colonial ambitions or not.

If strict $Z P G$ is observed, $L_{c}$ is of the order of the age of the : universe and it would be unlikely in the extreme for us to observe such a population. Variations in f would almost certainly increase the critical lifetime while variations in $\Psi$ will have an almost negligible effect. Therefore, strict ZPG readily explains our failure to.observe nearby extraterrestrial civilizations and we will confine our remaining considerations to cases including population growth and saturation.

Our conclusions for the case of density-dependent diffusion with population growth and saturation seem to be interestingly insensitive to the choice of input parameters. Reasonable variations in $f$, to allow for extensive colonization, 
changes $I_{c}$ by a factor 23 . We believe that $y$ camnot be much larger than $10^{-4} \mathrm{yr}^{-1}$ to be consistene with the very powerful population pressures which any colonial empire must have experienced earlier in its history than the cime of exrensive interstellar colonization. (In addition, one possible means of interstellar transport -- the "generation.ship" in which a much later generation of colonists arrives than that which originally set out -- imposes an extremely strict regime of ZPG on the settlers.) $\Delta x^{2}$ might be as large as $10^{3} \mathrm{pc}^{2}$, meaning that the colonists must be in the practice of searching comoratively large volumes of space before establishing a colony. ¥ conceivably could be much larger than $10^{-8} \mathrm{yt}^{-1}$, although values as large as those. typical for the European colonizacion of North America in the eighteenth century seem prohibitive for interstellar spaceFlight. But, even adopting $\Delta x^{2}=10^{3} \mathrm{pc}^{2}$ and $y=3 \times 10^{-4} \mathrm{ys}^{-1}$ we find that $I_{c} \sim 10^{5}$.ỵ. Even with massive interstellar colonization efforts, involving $10 \%$ of the base population every century, the Earth would not have been visited unless the colonizing civilization, were very long-lived. 
The effective velocity of the colonization wavefront is given by

$$
\mathrm{v}=1.2 \times 10^{-6}\left[\left(\gamma / 10^{-4}\right)\left(\Psi / 10^{-8}\right)\left(\Delta \mathrm{x}^{2} / 10\right)\right]^{\frac{1}{2}} \mathrm{pc}: \mathrm{yr}^{-1} \text {. }
$$

Note from dimensional analysis that this is the only combination of $\gamma, \Delta x^{2}$, and. $\Psi$ which will make a velocity. With nominal values of $\gamma, \Delta x^{2}$ and $\Psi$, the colonization wave-

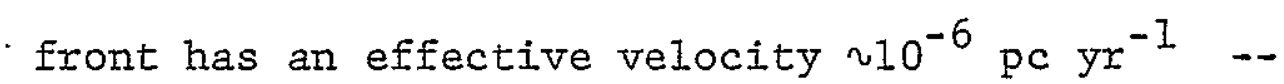
considerably slower even than the interstellar velocities of the Pioneer 10 and 11 and Voyager 1 and 2 spacecraft. 
Civilizations with lifecimes $i 10^{9}$ to $10^{10} \mathrm{yr}$, on the other hand, could have colonized the. Earth by now even if they originate some considerably greater distance Irom us within the Milky Way Galaxy: However, for: reasons which we have already mentioned -- dealing with the evolution of such very advanced societies -- we believe that their motivations for colonization may have altered utterly, and that their science and technology may be excremely different from anything we can recognize or even imagine.

We have found that only technical civilizations with lifetimes $\sim 10^{5}$ to $3 \times 10^{7}$ years could have initiated a colonial wavefront which has reached the Earth from the nearest technical civilization. The lifetimes of extraterrestrial technical. civilizations are, of course, highly uncertain but many workers in this field have hypothesized that only a tiny fraction of civilizations survive beyond $10^{5}$ to $10^{7}$ years (see, for example, Shklovskii and Sagan, 1966). In addition, there is the question of phase relations in the evolution of technical civilizations. In the solar neighborhood there are unlikely to be many scars with habitable planets which are significantly older than the sun, and we certainly expect a substantial waiting 
time -- perhaps $\sim$ several times $10^{9}$ yr -- for the indigenous evolution of technical societies. There are unlikely to be any colonizing civilizations in the solar neighborhood >>3 $\times 10^{7} \mathrm{yr}$ old.

It may be useful to divide technical civilizations into two categories: young $\left(<<10^{6} \mathrm{yr}-01 \mathrm{~d}\right)$ and $01 \mathrm{~d}\left(>>10^{6}\right.$ yx old). 01d civilizations are unlikely to be colonial powers in the sense we are describing. Young civilizations may be embarked on extensive colonial ventures but, compared to the volume of the Milky Way, these are of very limited scope. There may be empires of tens or even hundreds of thousands of worlds. But it is implausible that a Galactic Empire exists: there are simply too many worlds to conquer. .

The number of habitable planets in the Galaxy may exceed the number of individuals on the Earth. Might the political evolution of the Galaxy to some extent parallel that of the Earth, in which individuals start out more or less alone, and eventually form larger family group.s, tribes, city/states, nations, super-powers, and, perhaps eventually, a single global state? In that case the Earth is at the present time still in the situation of a lone individual -- 
one who even wonders if there are any other people at all. The stage we have been describing in this paper, when there are tens or hundreds of thousands. of colonial worlds for each interstellar civilization, corresponds to a much later stage in human history, roughly that of the first city/states. It is only at this point that groups interact. Thus Star Wars, if there are any, occur at the level of city/states -- Athens versus Sparta.

It is interesting to speculate on the likelihood of such encounters. For a random, uniform distribution of civilizations the probability $p$ of an interaction would behave as

$$
p \leq(d / \Lambda)^{3}
$$

wherein is the mean distance between civilizations. [eq. (76)] and $d$ is the mean value of the maximum radius of an. interstellar empire, i.e.,

$$
d=V I=\widetilde{v}(D Y)^{\frac{1}{2}} I
$$

Using (76), (79), (83) and (84) we find

$$
p \leqslant\left(I / I_{c}\right)^{4}
$$

for $I<I_{C}$. Thus, the likelihood of two civilizations 
interacting is remote unless $L \sim I_{C}$. (This argument depends of course on a given civilization growing in a manner that is independent of its neighbors.)

\section{If the probability of Star Wars goes as $\mathrm{L}^{4}$; if} the canonical lifetime for contact to be just occurring is $10^{7}$ yrs; and if we were to imagine all civilizations at $I=10^{6} \mathrm{yrs}$, only 1 in $10^{4}$ civilizations will be interacting. Since $N=10^{6}, ~ v 100$ will be interacting at a given time. If $I=10^{5} \mathrm{yrs}$, then 1 in $10^{8}$ civilizations will be interacting and, for $\mathbb{N}<10^{6} \quad$ civilizations, there are no star Wars. Thus, if warfare is an illness which is outgrown in the first $10^{5}$ years or so of the existence of a galactic civilization then there never are interstellar conflicts.

The slow propagation speed of the colonization wavefront does not exclude specific exploratory missions to targets of particular astrophysical or biological interest. The wavefront of the nearest colonial civilization may be tens or even only a few pc from the Earth and able to visit our solar system in relatively short times in the likely case that starship velocities $\geq 0.1 \mathrm{c}$ exist. They have not visited the Earth because there is no motivation, no apparent 
way for a neighboring civilization to know of the existence of our developing interstellar civilization. This situation may change in the relatively near future. Military and astronomical radar systems and, especially, commercial television have been for the last several decades generating an unmistakable radio signature, now $10 \mathrm{pc}$ away, of the existence of an emergent technical society in the vicinity of the Sun. Depending on the disposition of nearby civilizations, their colonies and their exploratory vessels, and how carefully they are examining the radio spectrum in eavesdropping mode, the leakage radiation from our technology may stimulate an exploratory mission from the nearest galactic civilization within the next century or less. 
Acknowledgement

We are grateful to S. A. Levin of the Department of Ecology and Systematics, H. Kesten of the Department of Mathematics, R.V.E. Lovelace of the Department of Applied Physiç, and G. V. Chester of the Department of Physics, all at Cornell University; to C. Alcock, F. J. Dyson, and H. Woolf of the Institute for Advanced Study; to R. M. May of the Department of Zoology, Princeton University; and, especially, to Steven Soter of the Laboratory for Planetary Studies at Cornell University, for stimulating discussions. This research was supported by the Planetary Biology Division of the Nationa1 Aeronautics and Space Administration under Grant NGR 33-010-101 and by NSF grant PHY 77-2-612. 


\section{References}

Bal1, John A. (1973) The zoo hypothesis, Icarus 19, $347-349$.

Barenblatt, G.I. (1952) On some unsteady motions of

a liquid and a gas in a porous medium, Drik1.

Mat. Mekh. 16, 67-78. (In Russian)

Barenblatt, G.I. and Zel'dovich, Ya.B. (1972) Selí-

.... similar solutions as incermediate asymptocics, Ann: Rev: Fluid Mech: 4, 285-312.

Chandrasekhar, S. (1943) Stochestic problems in physics and astronomy, Rev. Mod. Phys. 15, 1-8.9.

- Coale, A.J. (1974) The history of the human population, Scientific American 23I, 12-2:5.

Cole, Lamont C. (1954) Population consequences of lifie history phenomena, Quarcerly Review of Biology 29, $103-137$.

Courant, ‥ and Friedrichs, K.0. (1948) Subersonic Flow and Shock Waves (New York: Interscience):

Crank, J. (1956) The Mathemacics of Diffusion (Oxford: Clarendon Press). 
Cox, I.J. (1976) Correspondence: an explanation for the absence of extraterrestrials on earth, Q. II R. Astr. Soc. 17, 201-208. Davis, H.T. (1962) Introdiction to Nonilinear Differential and Integral Equations (New York: Dover). Dole, Stephen H. (1964) Habitable Plianets for Man (New York: Blaisdell Publishing Company): Dyson, Freeman J. (1966) The search for extra-terrestrial technology, in Perspectives in Modern Physics; Essays in Honor of Hans Bethe on the Occasion of his Sixtieth Birthday, R.E. Marshak, ed. (New York: Interscience Publishers): Eisen, D. (1967) On the numerical solution of $u_{t}=u_{r r}+$ $\frac{2}{\mathrm{r}} u_{r}$, Num: Math. 10, 397-409.

Fisher, R.A. (1937) The wave of advance of advantageous genes, Ann. Eugen. I, 355-369.

Guderley, G. (1942) Starke kugelige und zylindrische Verdichtungsstösse in der Nähe des Kugelmittelpunktes bzw der Zylinderachse, Iuftiahrtforschung 19, 302-312. .Gurney, W.S.C. and Nisbet, R.M. (1975) The regulation of inhomogeneous populations, J.' Thieor. Bijo1. 52 $441-457$. 
Gurtin, M.I. and MacCamy, R.C. (1977) On the difiusion of biological populations, Mach. Biosci. 33, 35-49.

Hadeler, R.P. (1977) Nonlinear Diffusion Equacions in Biology, from proc. Conf. Partial

Differential Equations, Dundee, Scotiand, 1976. In

Lecture Noces in Mathematics (Heidelberg:

Springer-Varlag).

Hart, Michael H. (1975) An explanacion for che absence of extraterrestrials on Earth; ‥ JI R. Astro. Soc. 16, 128-135.

Harc, Michael (1978) The evolution of the atmosphere of the Earth; ' Icarus 33, 23-39.

Eart, Michael (1979) Habicable zones about main-sequencs scars, Icarus, in press:

Howard, W.E. (1960) Innace and environmental dispersal of individual vertebrates, Am. 플. Nat. 63, 152-151. Isazcman, R. and Sagan, Carl (I977) Computer simulations of planetary accretion dynamics: Sensitivicy to. initial conditions, Icarus 31, 510-533.

Jones, i.M. (1976) Colonization of the galaxy; Icazus 28, $421-422$.

Jones, 叉.M. (1978) Interste1lar colonization, J.B.I.S. 3I, $103-107$.

Karlin, S. and Taylor, H.M. (1975) A First Course in Stochastic Processas (New York: Academic).

Kevles, D. (1975) Private communication. 
Klass, Philip J. (1974) UFO's-- Explained (New York: Random House).

Knerr, B.F. (1977) The porous medium equation in one dimension, Trans. Amex. Math. Soc. 234, 381-415.

Kolmegoroff, A., Petrovsky, I. and Piscounoff, N. (I937) Etude de l'equation de la diffusion avec croissance de la quantite de matiere et son application a un probleme biologique, Bull. Univ. Moskou, Ser. Internat., Sec. A, 1, 非6, 1-25.

Ianger, W.L. (1964) The black death, Scientific American 210, $114-121$.

Lax, P.D. (1969) Nonlinear partial differential equations and computing, SIAM Rev. 11, 7-19.

Levin, S.A. (1976) Population dynamic models in hererogeneous environments, Am. Rev. Ecol. Syst. I, $287-310$.

Mcivurtrie, R. (1.978) Persistence and stability of ...single-species and prey-predator systems in ... spatialiy heterogeneous environments, Math. Biosci. 39, 11-51. 
Muskat, M. (1937) The Flow of thomogeneous Fluids Through Porous Media (New York: McGraw-HiII). Needham, Joseph (1971) Science end Civilization in China, Vo1. V.Part 2 (London: Cambridge Universicy Press). Noble, J.V. (1974) Geographical and temporal development of olagues, Nature 250;726-728.

O'Neill, Gerard'(1975) Space colonies and energy supply co the Earth, Science 190, 943-947.

O'Neil1, Gerard (1978) The High Frontier (New York: Bantam). Pattle, R.E. (1959) Difiusion Irom an instantaneous point source with a concentration-dependent coefficient, Q.J. Mech. Apo1. Math. 12, 407-409.

Philip, J.R. (1970) Flow in porous media, Ann. Rev. Fluid eeㄴ. 2. 177-204.

Potter, J. (1965) The growth of popularion in America, 17001860, in Population in History (D.V. Glass and - D. $\vec{E} . \dot{C}$. Eversley, eds.) (Nen York: Edward Arnold Publishers).

Richtmeyer, R.D. and Morton, K.W. (1967) Diffierence Methods For Inicial-Veine Problems (New York: Academic). Sagan, C, (1961) The planet Venus, Science, 133, 849-858. 
Sagan, C. (1963) Direct contact among galactic civilizations by relativistic interstellar spaceflight, Planet. Space Sci. 11, 485-498.

Sagan, C. (1973a) On the detectivity of advanced galactic civilizations, Icarus 19, 350-352.

Sagan, C. (1973b), ed. Communication with Extraterrestrial Intelligence (CETI) (Cambridge, Mass.: M.I.T. Press).

Sagan, C. (1973b) Planetary engineering on Mars, Icarus 20, $513-514$.

Sagan, C. (1974) An introduction to the problem of cosmic communication, in Cosmic Communication, C. Ponnamperuma and A.G.W. Cameron, eds. (Boston: Houghton Mifflin).

Sagan, C. (1979) Broca"s Brain (New York: Random House, in press). Sagan, C, and Page, T,, eds. (1972) UFO's: A Scientific Debate (Ithaca, N.Y. and London: Cornell University Press).

Sedov, I.I. (1959) Similarity and Dimensional Methods in Mechanics (New York: Academic Press).

Shklovskii, I.S. (1976) Could intelligent life in the universe be unique? Voorosy Filosofii 95, 80-93 (In - Russian).

Shklovskii, I.S. and Sagan, C、 (1966) Intelligent Life in the Universe (San Francisco: Holden-Day). 
Ske1law, J.G. (1951) Random dispersal in theoretical populations, Biometrika 38, 196-218.

Steele, J.H. (1974) Spatial heterogeneity and population stability, Narure 248, 83.

Story, Ron (1976) The Space-God's Revealed: A Close Look at the Theories of Erich von Daniken (New York: Harper and Row).

Von Hoener, Sebastian (1973) Population explosion and interscellar expansion (Green Bank, tiest Virginia:

Sational Radio Astronomical Observatory) Reprint Series B, No. 330. See also von Hoerner, in Sagan (1973b).

Zel'dovich, Ya.B. and Raizer, Yu.P. (I967) Physics of Shock waves and High-Temperature Hydrodynamic Phenomena, Vol. II, Chapter X (New York: Academic). 
Figure 1. Thermal wave profiles for diffusion coefficients with a power law density dependence.

Figure 2. Wave of advance of the Bubonic Plague.

Figure 3. Travelling wave front for three model equations. 


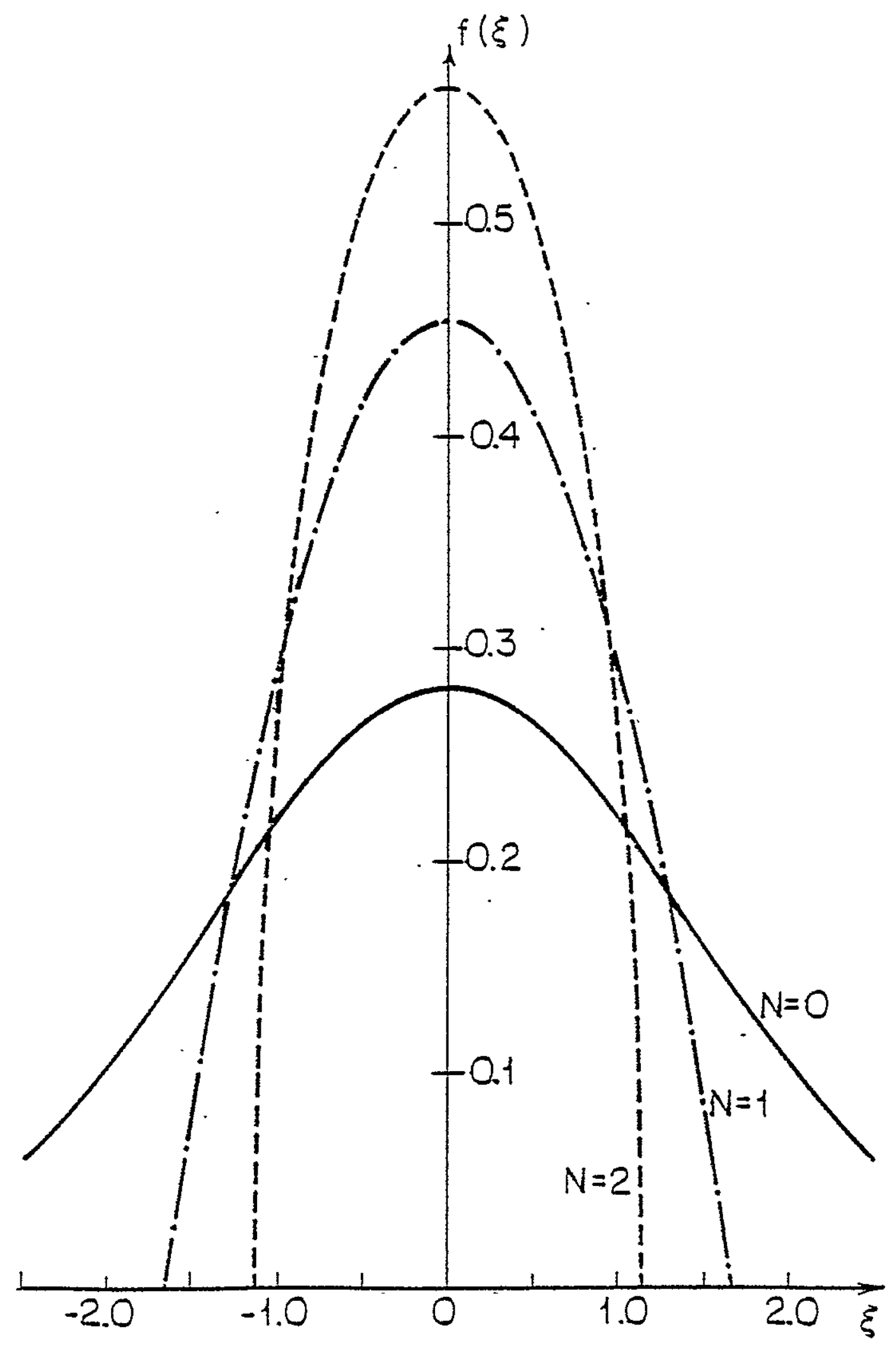

Figre 1 


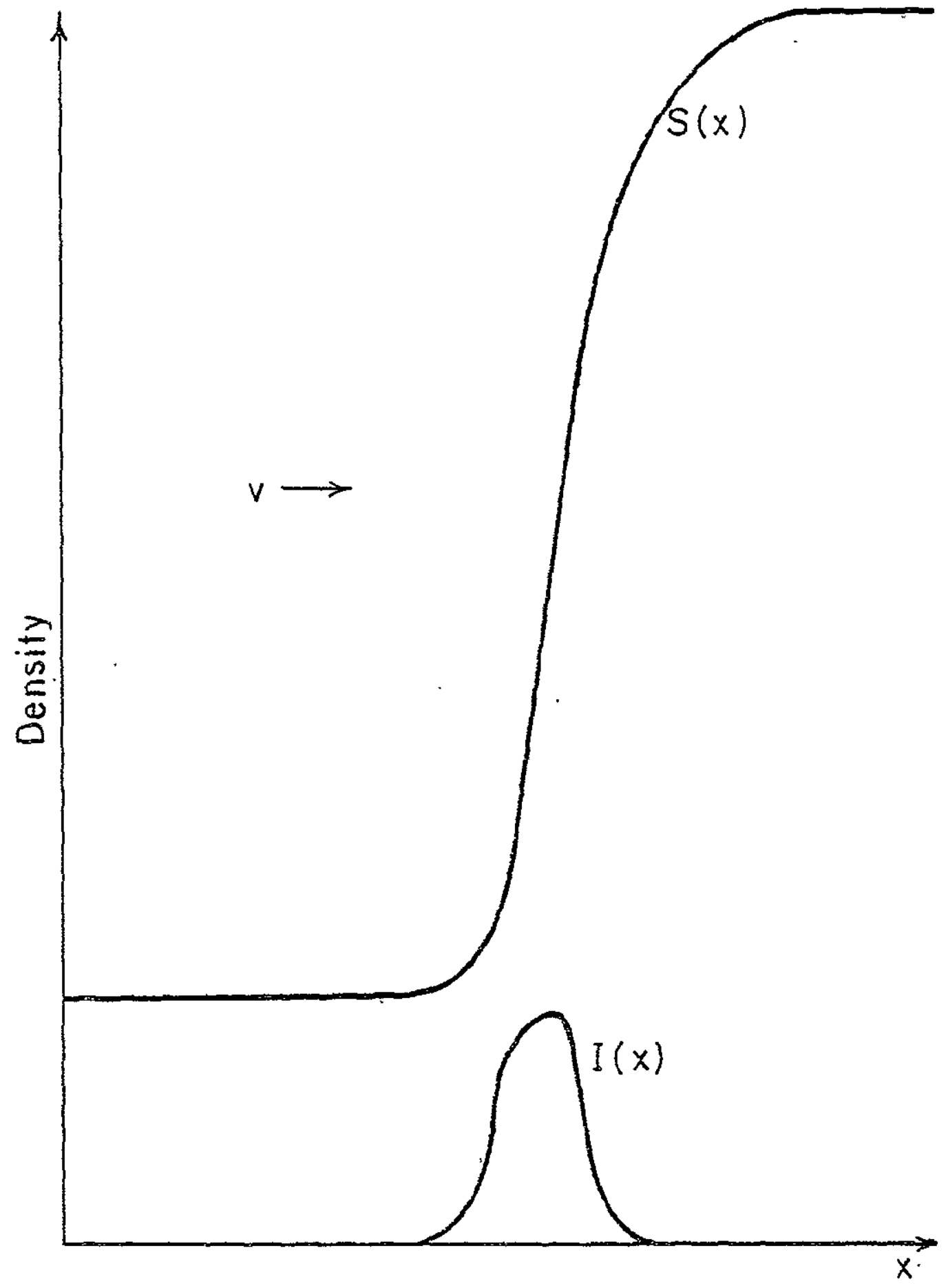

Figure 2 


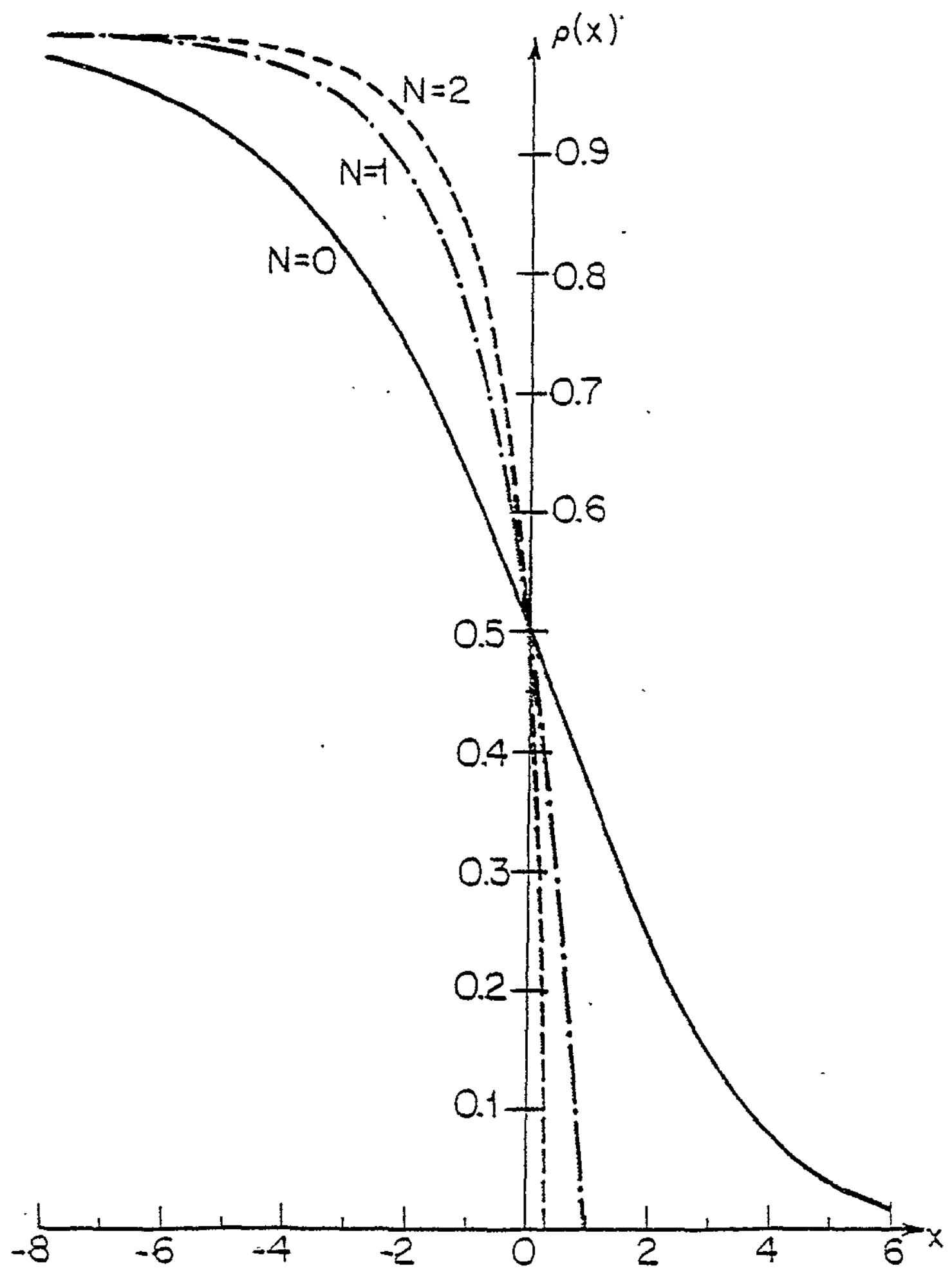

Finge 3 